\title{
Dynamical Analysis of the Circumprimary Planet in the Eccentric Binary System HD 59686
}

\author{
Trifon Trifonov ${ }^{1,2}$ (iD), Man Hoi Lee ${ }^{1,3}$ (D), Sabine Reffert ${ }^{4}$ (D), and Andreas Quirrenbach ${ }^{4}$ \\ ${ }^{1}$ Department of Earth Sciences, The University of Hong Kong, Pokfulam Road, Hong Kong; trifonov@mpia.de \\ ${ }_{2}^{2}$ Max-Planck-Institut für Astronomie, Königstuhl 17, D-69117 Heidelberg, Germany \\ ${ }^{3}$ Department of Physics, The University of Hong Kong, Pokfulam Road, Hong Kong \\ ${ }^{4}$ Landessternwarte, Zentrum für Astronomie der Universität Heidelberg, Königstuhl 12, D-69117 Heidelberg, Germany \\ Received 2017 April 3; revised 2018 February 28; accepted 2018 March 3; published 2018 March 29
}

\begin{abstract}
We present a detailed orbital and stability analysis of the HD 59686 binary-star planet system. HD 59686 is a single-lined, moderately close $\left(a_{B}=13.6 \mathrm{au}\right)$ eccentric $\left(e_{B}=0.73\right)$ binary, where the primary is an evolved $\mathrm{K}$ giant with mass $M=1.9 M_{\odot}$ and the secondary is a star with a minimum mass of $m_{B}=0.53 M_{\odot}$. Additionally, on the basis of precise radial velocity (RV) data, a Jovian planet with a minimum mass of $m_{p}=7 M_{\text {Jup }}$, orbiting the primary on a nearly circular S-type orbit with $e_{p}=0.05$ and $a_{p}=1.09$ au, has recently been announced. We investigate large sets of orbital fits consistent with HD 59686's RV data by applying bootstrap and systematic grid search techniques coupled with self-consistent dynamical fitting. We perform long-term dynamical integrations of these fits to constrain the permitted orbital configurations. We find that if the binary and the planet in this system have prograde and aligned coplanar orbits, there are narrow regions of stable orbital solutions locked in a secular apsidal alignment with the angle between the periapses, $\Delta \omega$, librating about $0^{\circ}$. We also test a large number of mutually inclined dynamical models in an attempt to constrain the three-dimensional orbital architecture. We find that for nearly coplanar and retrograde orbits with mutual inclination $145^{\circ} \lesssim \Delta i \leqslant 180^{\circ}$, the system is fully stable for a large range of orbital solutions.
\end{abstract}

Key words: planetary systems - planets and satellites: detection - planets and satellites: dynamical evolution and stability - techniques: radial velocities

\section{Introduction}

The first Doppler surveys looking for extrasolar planets were focused on finding solar system analogs and usually avoided known binary stars with semimajor axes $a_{B} \leqslant 200$ au (see Eggenberger \& Udry 2010; Thebault \& Haghighipour 2014). As a result, the number of known binary systems with planets orbiting around one of the components (in circumstellar or S-type orbits) or around both stars (in circumbinary or P-type orbits; see Dvorak 1986) is still relatively low when compared to planets orbiting single stars. To date, ${ }^{5}$ we know of $\sim 50$ S-type planets that are part of wide binaries separated by at least 50-1000 au (Roell et al. 2012) and 20 P-type planets orbiting both stars where the binary separation is below 1 au (mostly discovered with the Kepler satellite; Borucki et al. 2010; Doyle et al. 2011; Welsh et al. 2012; Kostov et al. 2013; Leung \& Lee 2013). However, only a handful of S-type planet candidates in moderately close binary systems $\left(a_{B} \leqslant 30 \mathrm{au}\right)$ are known in the literature, and they all were discovered using the radial velocity (RV) method.

A famous example is the $\gamma$ Cephei binary system, which consists of a K-giant primary of $M=1.6 M_{\odot}$ and a secondary star with a minimum mass of $m_{B} \sin i=0.44 M_{\odot}$ separated by $a_{B} \sim 19$ au. This system has a Jovian planet with a minimum mass of $m_{p} \sin i \sim 1.7 M_{\text {Jup }}$ (Campbell et al. 1988; Hatzes et al. 2003) orbiting on a stable orbit around the primary star at $a_{p} \sim 2.0$ au (Haghighipour 2006). A planet candidate on an S-type orbit is also evident in the RV data taken for the HD 196885 binary system (Correia et al. 2008). This system consists of an F8V primary of $M=1.3 M_{\odot}$ and a secondary

\footnotetext{
http://www.univie.ac.at/adg/schwarz/multiple.html
}

star with a minimum mass of $m_{B} \sin i=0.45 M_{\odot}$, orbital semimajor axis $a_{B}=21 \mathrm{au}$, and eccentricity $e_{B}=0.42$. The double Keplerian best fit for the RV data of HD 196885 reveals an S-type planet around the primary with $a_{p} \sim 2.6 \mathrm{au}$, $e_{p} \sim 0.48$, and a minimum mass of $m_{p} \sin i \sim 3.0 M_{\text {Jup }}$. Chauvin et al. (2011) carried out dynamical simulations that show that the planet's orbit is more stable in a highly inclined configuration near the equilibrium points of the Lidov-Kozai regime (Kozai 1962; Lidov 1962). Later, Giuppone et al. (2012) confirmed the stability of a highly inclined configuration with a mutual inclination of $\Delta i \approx 43^{\circ}$ or $137^{\circ}$, but they also found stable, nearly coplanar configurations, where the planet's orbit is either prograde or retrograde, with the retrograde orbits being less chaotic.

Another remarkable example is the $\nu$ Octantis binary (Ramm 2015; Ramm et al. 2009; 2016). This system consists of an $M=1.6 M_{\odot}$ K1 III giant primary and a low-mass secondary star separated by only $a_{B}=2.5$ au, with a moderate eccentricity of $e_{B}=0.24$. The binary inclination is well constrained at $i_{B}=71^{\circ}$, which yields a secondary mass of $m_{B}=0.6 M_{\odot}$. A lower-amplitude periodic RV variation is present in addition to the secondary-star RV signal, and if these variations are due to an orbiting planet, then the S-type companion would have $a_{p} \sim 1.2 \mathrm{au}, e_{p} \sim 0.1$, and a minimum mass of about $m_{p} \sin i \sim 2.0 M_{\text {Jup }}$. The planetary interpretation is problematic because the best-fit orbit (with semimajor axis ratio $a_{p} / a_{B} \approx 0.47$ ) is located well outside the boundary for stability if one assumes a coplanar and prograde planet with respect to the binary's orbit (Holman \& Wiegert 1999). However, Eberle \& Cuntz (2010) and Goździewski et al. (2013) showed that a nearly coplanar retrograde orbit is stable, even though the stable region is small due to nearby mean- 
motion resonances (MMRs) at the 2:1, 3:1, and 5:2 period ratios.

The existence of prograde, retrograde, or even Lidov-Kozai resonance S-type giant planets as a part of moderately compact systems remains a very challenging dynamical problem. Apart from the long-term stability problem, it is puzzling how planets can grow through core-accretion or disk-instability mechanisms in such close binaries. These systems provide important clues on how planets could form and remain in stable orbits around a star under the strong gravitational influence of a close stellar companion.

In this work, we study the HD 59686 single-lined binary system, which is composed of a $1.92 M_{\odot} \mathrm{K}$ giant and a lowmass star with a minimum mass of $m_{B} \sin i=0.53 M_{\odot}$. This system was reported to have a massive $\left(m_{p} \sin i \approx 7.0 M_{\text {Jup }}\right)$ Jovian S-type planet orbiting at $a_{p}=1.09$ au around the primary star (Ortiz et al. 2016). The binary itself, however, is very eccentric $\left(e_{B}=0.73\right)$, which challenges the planet's orbital stability. We carry out an extensive statistical and dynamical analysis of the available RV data to demonstrate that this system has stable configurations and further constrain its orbital parameters.

This paper is organized as follows. In Section 2, we review the physical configuration of the HD 59686 system. Section 3 describes the methodology of our dynamical fitting and longterm stability analysis. In Section 4, we introduce the best-fit results from our tests, and we reveal the possible S-type planet configurations. In Sections 5 and 6, we present dynamical and stability results around the best fits based on our bootstrap and systematic parameter grid search analysis. Finally, in Section 7, we present conclusions based on our results and discuss the possible S-type planet configurations.

\section{The HD 59686 Binary-planet System}

\subsection{System Configuration}

HD 59686 (=HR 2877, HIP 36616) is a bright, photometrically stable ( $V=5.45 \mathrm{mag}$; van Leeuwen 2007 ) horizontalbranch (HB) red giant star with an estimated mass of $M=1.92 \pm 0.21 M_{\odot}$, radius of $R=13.2 \pm 0.3 R_{\odot}$ (Reffert et al. 2015), and metal abundance of $[\mathrm{Fe} / \mathrm{H}]=0.15 \pm 0.1$ (Hekker \& Meléndez 2007). With luminosity $L=73.3 \pm 3.3 L_{\odot} \quad$ and effective temperature $T_{\text {eff }}=4658 \pm 24 \mathrm{~K}$, HD 59686 is a typical K2 III giant star. More physical parameters of HD 59686 can be found in Reffert et al. (2015).

Based on 88 precise $\left(5-8 \mathrm{~m} \mathrm{~s}^{-1}\right)$ RV observations of HD 59686 taken at Lick Observatory between 1999 November and 2011 December, Ortiz et al. (2016) reported that HD 59686 is actually part of a much more complicated threebody system. The Keplerian orbital solution for HD 59686 given in Ortiz et al. (2016) shows that the RV data have a largeamplitude variation of $K_{B}=4014.12 \pm 5.84 \mathrm{~m} \mathrm{~s}^{-1}$, whose characteristic RV shape reveals a stellar companion with $m_{B} \sin i=0.53 M_{\odot}$ on a highly eccentric orbit $\left(e_{B}=0.73\right)$. The large eccentricity and the fact that the binary recently passed through its periastron ( 2008 February) allowed the orbital period to be well determined as $P_{B}=11679.94 \pm 192.92$ days, even though the time span of the observations does not cover a full period of the binary orbit. In addition, the RV data yielded a lower-amplitude signal of $K_{p}=136.92 \pm 3.31 \mathrm{~m} \mathrm{~s}^{-1}$ with a derived period of
$P_{p}=299.36 \pm 0.28$ days. This signal is due to a Jovian planet with a minimum mass of $m_{p} \sin i=6.92 M_{\text {Jup }}$ on a nearly circular $\left(e_{p}=0.05 \pm 0.02\right)$ S-type orbit around the primary $\mathrm{K}$-giant star. The planetary signal remained coherent over many periods and was further confirmed by Trifonov et al. (2015) using follow-up RV measurements in the near-infrared taken with ESO's VLT spectrograph CRIRES (Kaeufl et al. 2004).

\subsection{Constraints on the Planetary Companion from the Hipparcos Intermediate Astrometric Data}

HD 59686 was a target of the Hipparcos mission (HIP 36616). We analyze the Hipparcos Intermediate Astrometric Data of HD 59686 based on the rereduction by van Leeuwen (2007) in the same way as described in Reffert \& Quirrenbach (2011). We ignore the stellar companion, since its period is much longer than the Hipparcos mission duration, and fit only the astrometric orbit of the planetary companion to the abscissa residuals, simultaneously allowing for adjustments in the standard astrometric parameters (position, proper motion, parallax) and keeping the spectroscopic parameters fixed. The best fit occurs at an inclination $i_{p}=2.9$ and longitude of the ascending node $\Omega_{p}=266^{\circ} .7$. The joint $3 \sigma$ confidence region extends from $i_{p}=1.6$ to $12^{\circ} .8$ and from $\Omega_{p}=206^{\circ} .8$ to $308^{\circ} .2$. Thus, a significant parameter range in $i_{p}$ and $\Omega_{p}$ can be formally rejected as a possible solution, and in Reffert \& Quirrenbach (2011), we argued that this is the best indicator for an actual detection of the astrometric orbit.

However, there are several concerns with the Hipparcos data of HD 59686.

(1) The reduced $\chi^{2}$ value in the van Leeuwen version of the Hipparcos catalog is 0.71 , already quite small. It indicates that either the errors are overestimated or that the solution is in fact already quite satisfactory, with no need for a better model for the measurements. The reduced $\chi^{2}$ value after fitting for the astrometric orbit is 0.59 , which is uncomfortably small.

(2) There is a clear correlation between the time of year and the scan direction (not only for HD 59686 but also for other targets). This is particularly a problem for periods close to $1 \mathrm{yr}$, which is the case for the planet orbiting HD 59686 (best-fit spectroscopic period $\approx 299$ days). On top of that, many Hipparcos measurements have been obtained during the same season, i.e., with the same scan direction. Thus, the Hipparcos measurements for HD 59686 are poorly constrained in the perpendicular direction (roughly coinciding with the right ascension direction) and can float freely to fit any astrometric orbit.

We believe that, as a result, the Hipparcos data for HD 59686 should be treated with caution. In fact, we will show later on that any solution with $i_{p}<30^{\circ}$ for the inner companion is highly improbable, so the detection of an astrometric orbit could not be brought in line with the observed $\mathrm{RV}$ data. We conclude that, most likely, the astrometric orbit of the inner companion has not been detected in the Hippar$\cos$ data.

\subsection{Dynamical Considerations}

As discussed in Ortiz et al. (2016), the stellar companion of HD 59686 must be either a low-mass (and low-luminosity) star, such as a $\mathrm{K}$ dwarf, or a white dwarf remnant. These scenarios are particularly important to trace the possible origin 
of the S-type planet. However, the question of whether the secondary is a $\mathrm{K}$ dwarf or white dwarf is of little importance for the goal of this paper, which is to study the current permitted (stable) orbital configuration.

At first look, it is unclear how the planet could remain stable in such a configuration. The binary semimajor axis is $a_{B}=13.6 \mathrm{au}$, but the pericenter distance is only $q_{B}=3.67 \mathrm{au}$, leading to strong interactions with the planet, which has $a_{p}=1.09$ and $q_{p}=1.03$ au. Assuming a minimum mass of $m_{B} \sin i=0.53 M_{\odot}$, the Hill radius of the secondary star can be approximated as

$$
r_{\mathrm{H}, B} \approx a_{B} \sqrt[3]{m_{B} / 3 M_{\star}} \approx 6.2 \mathrm{au},
$$

which would cover the S-type planet orbit entirely during the binary periastron passage. Due to the large eccentricity of the binary, however, one can define the Hill radius at the pericenter distance $q_{B}$ instead of $a_{B}$ (see Hamilton \& Burns 1992), which leads to a smaller value of $r_{\mathrm{H}, B} \approx 1.66 \mathrm{au}$. This suggests that the planet-secondary separation close to the binary periastron would be $\sim 1.5 r_{\mathrm{H}, B}$, making the survival of the planet still challenging.

A quick check using the empirical stability criterion of Holman \& Wiegert (1999) reveals that the critical (upper limit) semimajor axis for the S-type planet is $a_{\text {crit. }} \sim 1.03 \mathrm{au}$. Considering the binary-planet orbital uncertainties, we find that the S-type planet is most likely unstable, with an orbit slightly outside the stability region. Using similar empirical stability criteria from Eggleton \& Kiseleva (1995), we find that the planet is most likely stable, while the criterion of Mardling \& Aarseth (2001) suggests that the planet is unstable. In any case, these stability criteria agree that the planet is close to the stability border. Therefore, in this paper, we aim to inspect the three-dimensional orbital architecture of the HD 59686 system and study its long-term stability and dynamics.

\section{Methodology}

Our orbital analysis for HD 59686 is based on the multidimensional $\mathrm{N}$-body modeling scheme, which was previously applied to the 2:1 MMR exoplanet pairs around HD 82943 (Tan et al. 2013), HD 73526 (Wittenmyer et al. 2014), and $\eta$ Ceti (Trifonov et al. 2014). Briefly, we model the RV data using a Levenberg-Marquardt $\chi^{2}$ minimization scheme, which performs an $N$-body fit by integrating the equations of motion using the GraggBulirsch-Stoer integration method (see Press et al. 1992). The output parameters from our fitting code are the planetary and secondary-star RV semi-amplitude $\left(K_{p, B}\right)$, orbital period $\left(P_{p, B}\right), \quad$ eccentricity $\left(e_{p, B}\right), \quad$ argument of periastron $\left(\omega_{p, B}\right)$, mean anomaly $\left(M_{0 p, B}\right)$, inclination $\left(i_{p, B}\right)$ relative to the sky plane, and ascending node $\left(\Omega_{p, B}\right)$, as well as the $\mathrm{RV}$ offset $\left(\mathrm{RV}_{\text {off }}\right)$. All orbital parameters are the osculating ones in the Jacobi frame (e.g., Lee \& Peale 2003) at the first $\mathrm{RV}$ observational epoch, which is JD $=2,451,482.024$. Each fit comes with a reduced $\chi^{2}$ value $\left(\chi_{\nu}^{2}\right)$, the residual rms value, and the $1 \sigma$ uncertainties of the adjusted parameters obtained from the covariance matrix.

For HD 59686, we adopt a stellar mass of $1.92 M_{\odot}$ and a stellar velocity jitter amplitude of $20 \mathrm{~m} \mathrm{~s}^{-1}$. Ortiz et al. (2016) showed that the Lick data of HD 59686 are consistent with an additional stellar RV jitter of about $20 \mathrm{~m} \mathrm{~s}^{-1}$. The most likely reason for the notable $\mathrm{RV}$ noise in early $\mathrm{K}$ giants like
HD 59686 is solar-like $p$-mode oscillations (Barban et al. 2004; Zechmeister et al. 2008), which have typical periods much shorter than the typical time sampling of our Lick data and thus appear as scatter. Using the stellar parameters for HD 59686 from Reffert et al. (2015) and the scaling relation from Kjeldsen \& Bedding (2011), we estimated a jitter amplitude of $16.1 \pm 2.9 \mathrm{~m} \mathrm{~s}^{-1}$, which agrees well with the observed jitter of other K2 III giants in the Lick survey (Frink et al. 2001; Hekker et al. 2006; Trifonov et al. 2014; Reffert et al. 2015). Therefore, for our dynamical modeling, we adopt a uniform a priori stellar jitter value of $20 \mathrm{~m} \mathrm{~s}^{-1}$, which we quadratically $\operatorname{add}^{6}$ to the total RV data error budget.

All dynamical fits in our study are further tested for longterm dynamical stability. We integrate the orbits using a custom version of the Wisdom-Holman algorithm (Wisdom \& Holman 1991), modified to handle the evolution of hierarchical systems consisting of massive bodies (Lee \& Peale 2003). The bodies are assumed to be point masses, and mutual collisions between them are not considered in defining system stability. We also neglect general relativity and companion-star tidal effects during the simulations. We integrate the individual fits for a maximum of $10 \mathrm{Myr}$ by adopting an integration time step equal to 1 day. Our integration setup corresponds to more than $3 \times 10^{5}$ full binary orbits with about 300 steps per complete planetary orbit. We find that this setup is sufficient to resolve the planet's orbit with high resolution and study the system's long-term stability.

We define the HD 59686 system as stable if, during the integration, the companion bodies remain in orbits that do not deviate significantly from their initial best-fit configuration. The system's stability depends primarily on the survival of the S-type planet. In most cases, when the planet inclination is $30^{\circ}<i_{p}<90^{\circ}$, the planet has relatively low mass to perturb the binary orbit significantly and can be well approximated as a test particle in a two-body system. However, we also test fits with $i_{p}<30^{\circ}$, where the mass of the S-type body becomes quite large as $\sin i_{p}$ gets smaller, and thus it can significantly influence the binary orbit during the orbital evolution. A simulation is terminated and the system is considered unstable if at some point in the integration the semimajor axis $a_{p}$ or $a_{B}$ changes by more than $\pm 60 \%$ from the initial values or if $e_{p, B}>0.95$. Particularly, when $e_{p}>0.95$ and $a_{p}<1.2 \mathrm{au}$, the planet periastron distance to the central star, $q_{p}$, is well within the physical radius of the $\mathrm{K}$ giant $\left(q_{p}<R \approx 0.06 \mathrm{au}\right)$, and the planet will collide with the star. Although these criteria for instability are somewhat arbitrary, our simulations show that even small chaotic deviations in $a_{p}$ and $e_{p}$ quickly accumulate, and there are no cases where the orbits change significantly without exceeding these criteria.

\section{Best Fits \\ 4.1. Edge-on Prograde and Retrograde Fits}

The best coplanar and edge-on dynamical fit is generally consistent with the Keplerian fit shown in Ortiz et al. (2016). Our dynamical fit is close to a double Keplerian, since any significant gravitational perturbations on the planetary orbit

\footnotetext{
6 Alternatively, the RV jitter could be fitted as a free parameter of the RV model (e.g., Baluev 2009). The best double Keplerian fit of HD 59686 optimized with an additional jitter term to the Lick data yields a jitter value of $19.6_{-1.5}^{+1.8} \mathrm{~m} \mathrm{~s}^{-1}$, which is consistent with the uniform jitter value of $20 \mathrm{~m} \mathrm{~s}^{-1}$ adopted in this work.
} 
(and thus on the induced RVs) are expected to be detected only after a few binary cycles, while the RV data currently cover only $\sim 40 \%$ of one full binary orbit. We first keep the orbital inclinations fixed at $i_{p}=i_{B}=90^{\circ}$ and the difference between the lines of node $\Delta \Omega=\Omega_{p}-\Omega_{B}=0^{\circ}$, which defines a planar and prograde configuration. The best fit in this orbital configuration has $\chi_{\nu}^{2}=0.995$ and leads to orbital elements of $P_{p}=299.1 \pm 0.30$ days, $e_{p}=0.05 \pm 0.02, a_{p}=1.09 \mathrm{au}$, and mass of $m_{p}=6.97 M_{\mathrm{Jup}}$ for the planet and orbital elements of $P_{B}=11696.4 \pm 196.4 \quad$ days, $\quad e_{B}=0.73 \pm 0.003$, $a_{B}=13.61 \mathrm{au}$, and secondary-star mass of $m_{B}=558 M_{\mathrm{Jup}}$ for the binary. The full set of orbital elements and their bootstrap (see Section 5) and covariance matrix estimated uncertainties are given in Table 1, while the actual fit to the data (black curve in the upper panel) and its residuals are illustrated in Figure 1. The long-term evolution of the orbital semimajor axes and eccentricities is shown in Figure 3 (left panel). According to our stability criteria, this fit is stable for only about $42 \mathrm{kyr}$ before the planet collides with the star. A close examination of this fit indicates that the orbits show large variations in $e_{p}$ and small but chaotic variations in $e_{B}$. Eventually, the secondary companion excites the planet eccentricity above $e_{p}>0.95$, which interrupts our integration.

Since the best coplanar and prograde fit is unstable, we test how the fit quality and stability change if we allow noncoplanar orbits. We simplify this test by keeping the binary on an edge-on orbit with fixed $i_{B}=90^{\circ}$ and $\Omega_{B}=0^{\circ}$. For the planet, we also fix the inclination at $i_{p}=90^{\circ}$, but we systematically vary $\Omega_{p}$ between $0^{\circ}$ and $359^{\circ}$ with a step of $1^{\circ}$. Thus, in this test, we keep the companion masses at their minimum, while the mutual inclination comes only from the difference between the longitudes of the ascending nodes $\Delta \Omega=\Omega_{p}-\Omega_{B}$ following the expression

$$
\Delta i=\arccos \left[\cos \left(i_{p}\right) \cos \left(i_{B}\right)+\sin \left(i_{p}\right) \sin \left(i_{B}\right) \cos (\Delta \Omega)\right] .
$$

Figure 2 shows the results from this test. We plot the quality of the mutually inclined fit in terms of $\chi_{\nu}^{2}\left(\chi^{2}\right)$ as a function of $\Delta \Omega$ $(\Delta i)$. Horizontal dashed lines show the $1 \sigma, 2 \sigma$, and $3 \sigma$ confidence levels according to $\Delta \chi^{2}$. The best fit in Figure 2 appears at $\Delta i=180^{\circ}$, which is again a coplanar but retrograde planet orbit. The best coplanar prograde fit has $\chi_{\mathrm{CP}}^{2}=76.61$, while the best coplanar retrograde fit has $\chi_{\mathrm{CR}}^{2}=75.63$, resulting in $\Delta \chi^{2}=0.98$. This difference is slightly below the $1 \sigma$ limit, and thus the retrograde fit does not represent a significant improvement to our model. In Figure 2, most of the edge-on fits with $\Delta i<145^{\circ}$ are above $1 \sigma$ from the best fit and are unstable (red line), while all fits with $\Delta i$ between $145^{\circ}$ and $180^{\circ}$ are within $1 \sigma$ and are stable (thick blue line) for at least 10 Myr.

Figure 3 (middle panel) shows an $\sim 50$ kyr time span of the orbital evolution for the best coplanar retrograde fit. The semimajor axes $a_{p}$ and $a_{B}$ are nearly constant during the stability test. The planet eccentricity $e_{p}$ oscillates with a large amplitude between 0 and 0.35 , but the system remains stable, with the bodies well separated from each other. Interestingly, the mean period ratio of this stable retrograde fit is $P_{B} / P_{p} \approx 39$, but the system is not in 39:1 MMR, as none of the resonance angles associated with the 39:1 MMR are librating. For the $n: 1$ MMR, the resonance angles are

$$
\theta_{m=1, n}=\lambda_{p}-n \lambda_{B}+(m-1) \varpi_{p}-(m-n) \varpi_{B},
$$

Table 1

HD 59686 System Best Dynamical Fits

\begin{tabular}{|c|c|c|}
\hline \multicolumn{3}{|c|}{ Coplanar Edge-on Retrograde } \\
\hline Parameter & HD $59686 \mathrm{Ab}$ & HD 59686 B \\
\hline $\begin{array}{l}K\left[\mathrm{~m} \mathrm{~s}^{-1}\right] \\
P[\text { days }] \\
e \\
\omega[\mathrm{deg}] \\
M_{0}[\mathrm{deg}] \\
\mathrm{RV}_{\text {off }}\left[\mathrm{m} \mathrm{s}^{-1}\right] \\
i \text { [deg] } \\
\Omega[\mathrm{deg}] \\
\Delta i[\mathrm{deg}] \\
a[\mathrm{au}] \\
m\left[M_{\mathrm{Jup}}\right] \\
\mathrm{rms}\left[\mathrm{m} \mathrm{s}^{-1}\right] \\
\chi^{2} \\
\chi_{\nu}^{2}\end{array}$ & $\begin{array}{c}136.7_{-4.4}^{+3.7}( \pm 3.3) \\
299.0_{-0.3}^{+0.3}( \pm 0.3) \\
0.05_{-0.02}^{+0.03}( \pm 0.02) \\
126.8_{-24.5}^{+27.1}( \pm 28.3) \\
293.5_{-51.5}^{+29.5}( \pm 28.4) \\
247.1_{-9.3}^{+13.6}( \pm 12.4) \\
90.0^{\mathrm{a}} \\
180.0^{\mathrm{a}} \\
180.0 \\
1.089_{-0.001}^{+0.001} \\
6.96_{-0.23}^{+0.18} \\
19.46 \\
75.63 \\
0.982\end{array}$ & $\begin{array}{c}4013.7_{-7.7}^{+9.8}( \pm 20.5) \\
11669.3_{-147.0}^{+218.1}( \pm 194.7) \\
0.729_{-0.003}^{+0.004}( \pm 0.003) \\
149.4_{-0.2}^{+0.2}( \pm 0.1) \\
258.9_{-1.1}^{+2.6}( \pm 1.7) \\
\ldots \\
90.0^{\mathrm{a}} \\
0.0^{\mathrm{a}} \\
\ldots \\
13.591_{-0.145}^{+0.169} \\
558.46_{-0.91}^{+1.21} \\
\ldots \\
\ldots \\
\ldots\end{array}$ \\
\hline \multicolumn{3}{|c|}{ Mutually Inclined } \\
\hline Parameter & HD $59686 \mathrm{Ab}$ & HD 59686 B \\
\hline $\begin{array}{l}K\left[\mathrm{~m} \mathrm{~s}^{-1}\right] \\
P[\text { days }] \\
e \\
\omega[\mathrm{deg}] \\
M_{0}[\mathrm{deg}] \\
\mathrm{RV}_{\text {off }}\left[\mathrm{m} \mathrm{s}^{-1}\right] \\
i[\mathrm{deg}] \\
\Omega[\mathrm{deg}]\end{array}$ & $\begin{array}{c}130.1_{-3.0}^{+3.1}( \pm 26.2) \\
300.5_{-0.6}^{+0.2}( \pm 0.5) \\
0.08_{-0.02}^{+0.02}( \pm 0.02) \\
145.2_{-17.6}^{+19.2}( \pm 19.7) \\
280.4_{-19.9}^{+26.3}( \pm 20.0) \\
239.5_{-9.0}^{+13.0}( \pm 12.7) \\
178.8_{-0.5}^{+0.3}( \pm 0.3) \\
316.5_{-17.7}^{+10.4}( \pm 10.3)\end{array}$ & $\begin{array}{c}4020.0_{-5.5}^{+6.2}( \pm 182.1) \\
11398.3_{-95.2}^{+204.0}( \pm 1244.3) \\
0.725_{-0.002}^{+0.003}( \pm 0.003) \\
149.8_{-0.2}^{+0.2}( \pm 4.7) \\
256.4_{-0.7}^{+2.4}( \pm 21.0) \\
\ldots \\
86.4_{-2.4}^{+2.4}( \pm 3.3) \\
0.0^{\mathrm{a}}\end{array}$ \\
\hline $\begin{array}{l}\Delta i[\mathrm{deg}] \\
a[\mathrm{au}] \\
m\left[M_{\mathrm{Jup}}\right]\end{array}$ & $\begin{array}{c}92.73 \\
1.15_{-0.02}^{+0.02} \\
359.22_{-120.44}^{+114.33}\end{array}$ & $\begin{array}{c}\cdots \\
14.06_{-0.18}^{+0.26} \\
618.36_{-15.82}^{+18.45}\end{array}$ \\
\hline $\begin{array}{l}\mathrm{rms}\left[\mathrm{m} \mathrm{s}^{-1}\right] \\
\chi^{2} \\
\chi_{\nu}^{2}\end{array}$ & $\begin{array}{l}16.62 \\
55.50 \\
0.750\end{array}$ & $\begin{array}{l}\ldots \\
\ldots \\
\ldots\end{array}$ \\
\hline
\end{tabular}

\begin{tabular}{lcc}
\hline \hline & Coplanar Edge-on Prograde \\
\hline Parameter & HD 59686 Ab & HD 59686 B \\
\hline$K\left[\mathrm{~m} \mathrm{~s}^{-1}\right]$ & $137.0_{-4.5}^{+3.6}( \pm 3.4)$ & $4012.6_{-8.2}^{+9.9}( \pm 20.6)$ \\
$P[$ days $]$ & $299.1_{-0.3}^{+0.3}( \pm 0.3)$ & $11696.4_{-170.7}^{+209.2}( \pm 196.4)$ \\
$e$ & $0.05_{-0.02}^{+0.03}( \pm 0.02)$ & $0.730_{-0.003}^{+0.004}( \pm 0.003)$ \\
$\omega[\mathrm{deg}]$ & $121.1_{-25.7}^{+28.5}( \pm 28.7)$ & $149.4_{-0.1}^{+0.2}( \pm 0.1)$ \\
$M_{0}[\mathrm{deg}]$ & $299.5_{-32.7}^{+20.9}( \pm 28.4)$ & $259.2_{-1.5}^{+1.7}( \pm 1.7)$ \\
$\mathrm{RV}_{\text {off }}\left[\mathrm{m} \mathrm{s}^{-1}\right]$ & $248.6_{-10.4}^{+13.5}( \pm 12.5)$ & $\ldots$ \\
$i[\mathrm{deg}]$ & $90.0^{\mathrm{a}}$ & $90.0^{\mathrm{a}}$ \\
$\Omega[\mathrm{deg}]$ & $0.0^{\mathrm{a}}$ & $0.0^{\mathrm{a}}$ \\
$\Delta i[\mathrm{deg}]$ & 0.0 & $\ldots$ \\
$a[\mathrm{au}]$ & $1.089_{-0.001}^{+0.001}$ & $13.611_{-0.132}^{+0.163}$ \\
$m\left[M_{\mathrm{Jup}}\right]$ & $6.97_{-0.23}^{+0.18}$ & $558.41_{-0.99}^{+1.20}$ \\
$\mathrm{rms}\left[\mathrm{m} \mathrm{s}{ }^{-1}\right]$ & 19.59 & $\ldots$ \\
$\chi^{2}$ & 76.61 & $\ldots$ \\
$\chi_{\nu}^{2}$ & 0.995 & $\cdots$ \\
\hline
\end{tabular}

Note. Included are ${ }_{-0.0}^{+0.0}$ bootstrap uncertainties and $( \pm 0.0)$ covariance matrix uncertainties.

${ }^{\mathrm{a}}$ Fixed parameters.

where $n$ is positive for prograde motion and negative for retrograde motion, $\varpi_{p, B}$ are the longitudes of periastron, and $\lambda_{p, B}$ are the mean longitudes. All fits with $\Delta i$ between $145^{\circ}$ and $180^{\circ}$ have similar behavior for $a_{p}, a_{B}, e_{p}$, and $e_{B}$, while $\Delta i$ 


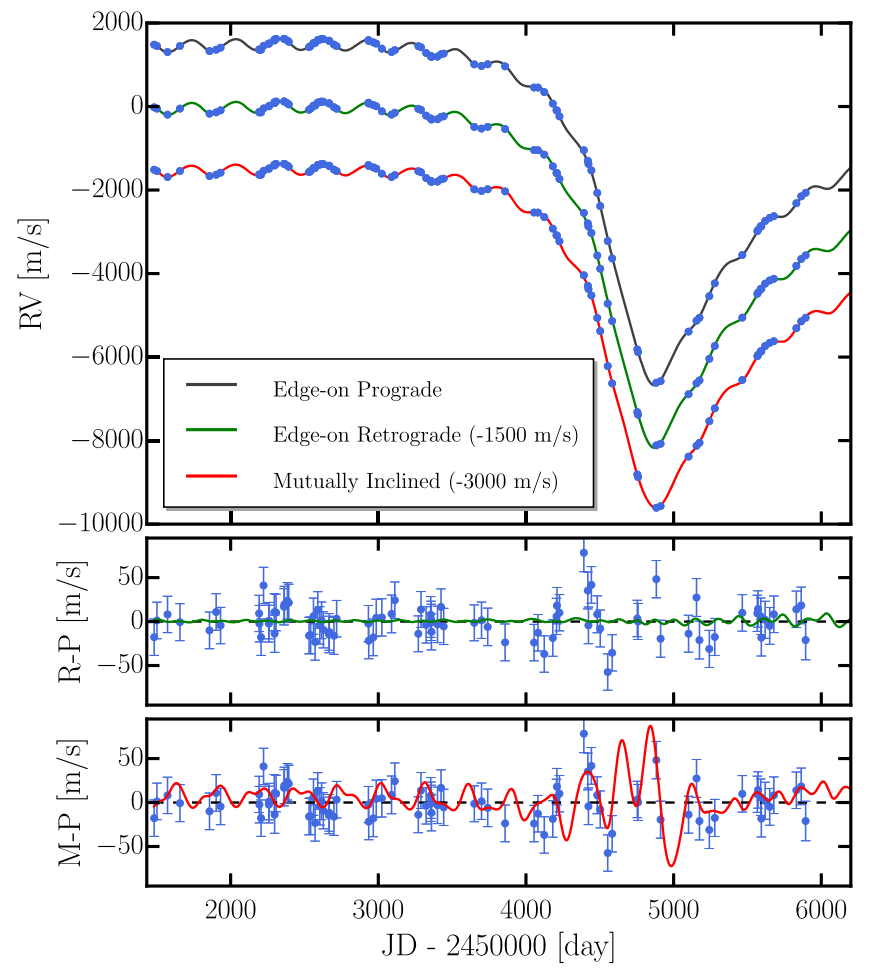

Figure 1. Three best-fit models to the Lick data (blue points). In the upper panel, the top curve (black) is the best edge-on coplanar prograde fit, while the middle and bottom curves are the best edge-on coplanar retrograde (green) and mutually inclined (red) fits, offset vertically for illustration purposes by -1500 and $-3000 \mathrm{~m} \mathrm{~s}^{-1}$, respectively. Error bars include $20 \mathrm{~m} \mathrm{~s}^{-1}$ added quadratically to the formal uncertainties to account for stellar jitter. The residuals of the best edge-on prograde fit are compared to the difference between the prograde fit and the best edge-on retrograde or mutually inclined fits in the lower two panels. The difference between the prograde and retrograde fits is very small. The mutually inclined fit better models some data points with large residuals in the other orbital fits, although these data points lie in the relatively sparsely sampled epochs around the periastron passage of the binary orbit.

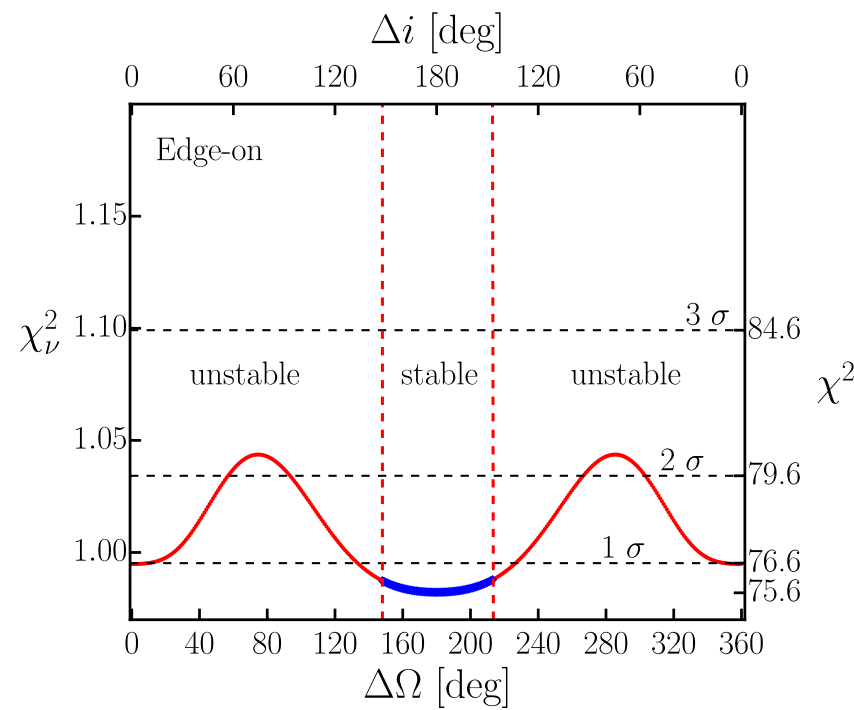

Figure 2. Edge-on $\left(i_{p}=i_{B}=90^{\circ}\right)$ but mutually inclined fits of HD 59686 . The mutual inclination angle $\Delta i$ in edge-on orbits comes from $\Delta \Omega=\Omega_{p}-\Omega_{B}$. The $\Delta \chi^{2}$ confidence levels in terms of $1 \sigma, 2 \sigma$, and $3 \sigma$ are drawn for the $\chi^{2}$ minimum, which is at edge-on and retrograde orbits $\left(\Delta i=180^{\circ}\right.$ ). All fits between $\Delta i \sim 145^{\circ}$ and $180^{\circ}$ (thick blue line) are stable. These fits also have better quality when compared to the prograde, polar, and near-polar fits, which are unstable. oscillates with small amplitude around the initial fitted value. None of them seem to be locked in an MMR.

The most likely reason for the wider stable region for the retrograde orbits is that the individual MMRs are of higher order for retrograde than prograde orbits. As demonstrated in Morais \& Giuppone (2012), at the same $n: q$ mean-motion ratio, the MMR is of order $n-q$ for prograde versus order $n+q$ for retrograde orbits, which, for the latter, results in much narrower MMR libration widths and thus smaller phase-space overlap of neighboring MMRs where the planet would likely be unstable. The findings of Morais \& Giuppone (2012), however, were restricted to the dynamics of S-type planets in circular binary systems, while the dynamics of the planet in the highly eccentric HD 59686 binary is far more complex. A more detailed analysis of resonance width and overlap for prograde and retrograde orbits using the formalism developed by Mardling (2008) in the context of the HD 59686 system will be presented in a future paper (K. H. Wong \& M. H. Lee 2018, in preparation).

\subsection{Inclined Coplanar Fits-Constraining $\sin i$}

Both prograde and retrograde edge-on best fits suggest a coplanar configuration. Therefore, as a next step, we test how the fit quality and stability for both configurations depends on the inclination $i$ (measured from the plane of the sky). For the prograde geometry, we fix $\Delta \Omega=0^{\circ}$ and $i_{p}=i_{B}$. We systematically vary $i_{p}$ and $i_{B}$ from $90^{\circ}$ to $5^{\circ}$ with a decreasing step of $1^{\circ}$, and thus we gradually increase the companion masses by a factor of approximately $\sin i$. The same test is done for the retrograde fits, which are constructed by keeping $\Delta \Omega=180^{\circ}$, $i_{B}=180^{\circ}-i_{p}$, and varying $i_{p}$ from $90^{\circ}$ to $5^{\circ}$ with a step of $1^{\circ}$. According to Equation (2), the prograde fits have a mutual inclination of $\Delta i=0^{\circ}$ and the retrograde fits $\Delta i=180^{\circ}$.

The results from this systematic test are illustrated in Figure 4, which shows a comparison between prograde and retrograde dynamical fits as a function of $\sin i$. The $\chi_{\nu}^{2}$ minimum for both prograde and retrograde cases is at $\sin i=1$, which corresponds to the same best fits presented in Table 1 and Figure 2. The $\Delta \chi^{2}$ confidence levels $(1 \sigma, 2 \sigma$, and $3 \sigma)$ in Figure 4 are measured from the best retrograde fit and represent the same confidence levels as in Figure 2. Clearly, our $N$-body fits can only weakly constrain the orbital inclination from the $\mathrm{RV}$ data. Overall, the retrograde fits have better $\chi_{\nu}^{2}$ values, but in both configurations, the fits are gradually becoming worse for lower $\sin i$ and thus higher planet and secondary-star masses. For retrograde fits down to $2 \sigma$, the orbital inclination can be between $10^{\circ}$ and $90^{\circ}$, while for prograde fits, the inclination can be between $20^{\circ}$ and $90^{\circ}$. In both configurations, however, the inclination is unlikely to be less than $30^{\circ}$, as the secondary star would then be at least a G-type main-sequence star with about twice the minimum mass. Such a stellar companion should have been detected by Ortiz et al. (2016) via the Large Binocular Telescope (LBT) angular differential imaging, but since it was not, we assume $\sin i_{B}=0.5$ as a lower limit.

When it comes to stability, all retrograde coplanar fits in Figure 4 are stable for $10 \mathrm{Myr}$, including those at very low inclinations, while none of the prograde fits are long-term stable. 

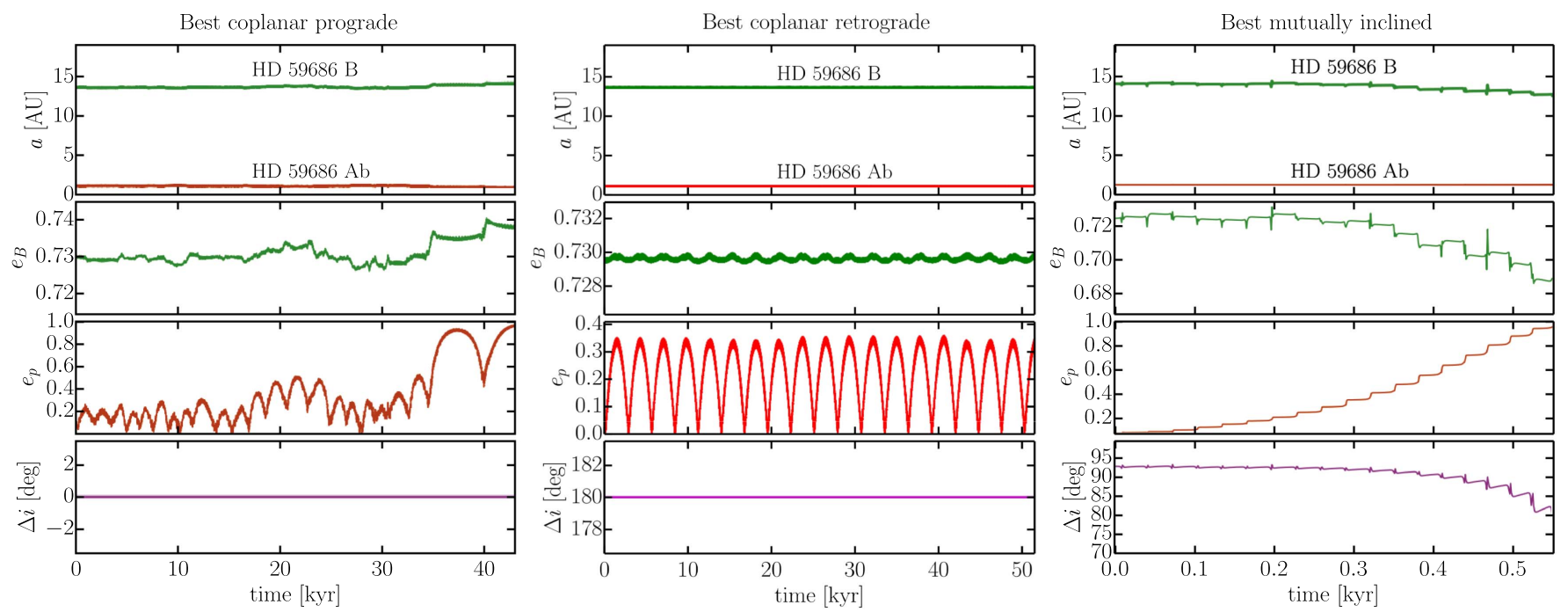

Figure 3. Semimajor axes, eccentricities, and mutual inclination evolution for the best coplanar edge-on prograde and retrograde fits and the best mutually inclined fit. The best coplanar prograde fit (left) is unstable on short timescales of about $43 \mathrm{kyr}$, when the planet eccentricity is excited to $e_{p}>0.95$, leading to collision with the star. The retrograde fit (middle) is stable during the $10 \mathrm{Myr}$ test ( $\sim 50 \mathrm{kyr}$ shown). In this fit, $e_{p}$ oscillates with large amplitude between 0 and 0.35 , but the orbits remain well separated and stable. The best mutually inclined configuration (right) does not survive even $600 \mathrm{yr}$. The planet is initially on a nearly circular orbit but with $\Delta i \approx 93^{\circ}$ with respect to the binary plane. Due to the Lidov-Kozai effect, the planet eccentricity is quickly excited to $e_{p}>0.95$.

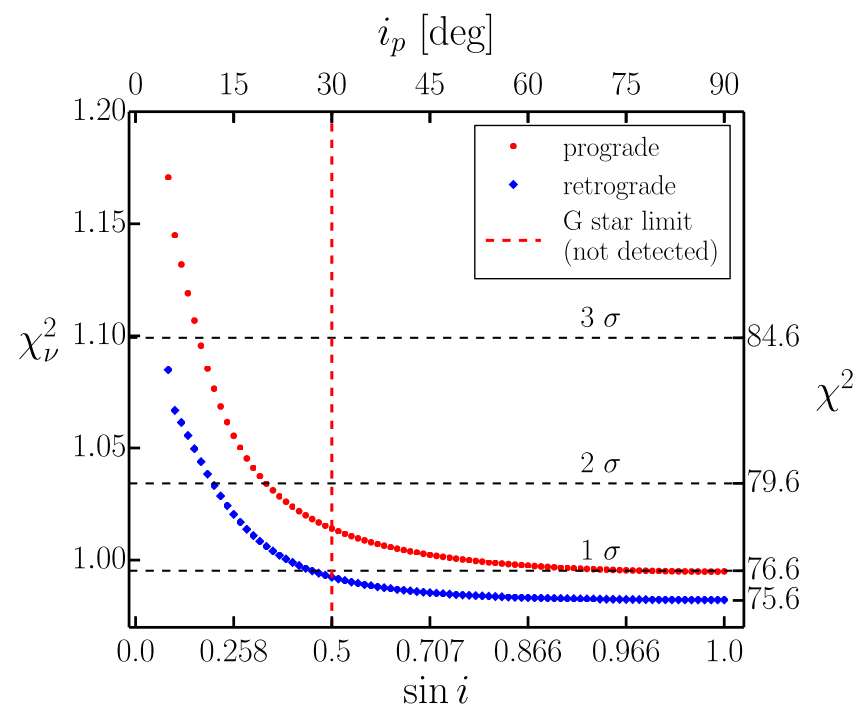

Figure 4. Comparison of the quality of coplanar prograde and retrograde fits as a function of the inclination $i$. Retrograde configurations have better $\chi_{\nu}^{2}$ than prograde ones and are all stable for $10 \mathrm{Myr}$, while none of the prograde fits are long-term stable. In both cases, $\chi_{\nu}^{2}$ is minimum at $i=90^{\circ}$ (edge-on) and increases only slowly with decreasing $i$. The $\Delta \chi^{2}$ confidence levels $(1 \sigma, 2 \sigma$, and $3 \sigma$ ) are obtained from the best retrograde fit, as in Figure 2. The red dashed line marks $i=30^{\circ}$, below which the secondary must be at least a $\mathrm{G}$ dwarf mass star, which would have been detected with LBT angular differential imaging (Ortiz et al. 2016).

\subsection{Mutually Inclined Fits-The Global Minimum}

Finally, in our dynamical modeling of HD 59686's RV data, we allow non-edge-on mutually inclined orbits by independently fitting $i_{p}$ and $i_{B}$ in the range between $0^{\circ}$ and $180^{\circ}$ and $\Delta \Omega$ between $0^{\circ}$ and $360^{\circ}$. In this way, we allow our fits to adopt a large range of companion masses, and we cover all possible orbital alignments.

Our mutually inclined best fit has a strong minimum with $\chi_{\nu}^{2}=0.75, \quad$ yielding $\quad P_{p}=300.5 \pm 0.5 \quad$ days, $e_{p}=0.08 \pm 0.02$, and $a_{p}=1.15$ au for the inner companion and $P_{B}=11398.3 \pm 1244.3$ days, $e_{B}=0.725 \pm 0.003$, and
$a_{B}=14.06$ au for the outer companion (see Table 1). Remarkably, this fit suggests that the inner companion has a nearly face-on orbit with well-constrained $i_{p}=178^{\circ} .8 \pm 0.3$. This means that the inner companion is no longer a planet but a stellar-mass companion with $m_{p}=359 M_{\text {Jup }} \quad\left(=0.34 M_{\odot}\right)$ forming an inner binary pair with the $\mathrm{K}$ giant. The outer companion has a nearly edge-on orbit with $i_{B}=86^{\circ} .4 \pm 3^{\circ} .3$ and mass $m_{B}=618 M_{\text {Jup }}\left(=0.59 M_{\odot}\right)$. The difference between the ascending nodes is $\Delta \Omega=316^{\circ} .5 \pm 10^{\circ} .3$, and according to Equation (2), this leads to $\Delta i=92^{\circ} .7 \pm 3.3$. We achieve practically the same fit (within the errors) with $\chi_{\nu}^{2}=0.75$ at $i_{p}=1.15 \pm 0.6, i_{B}=93^{\circ} .4 \pm 2.5$, and $\Delta \Omega=43^{\circ} .7 \pm 12^{\circ} .3$, which is a mirror image of the above orbital configuration.

The $\chi^{2}$ value for this mutually inclined fit is 55.5, which is much lower than the best coplanar retrograde fit with $\chi_{\mathrm{CR}}^{2}=75.6$. This fit, however, has three additional fitting parameters compared to the edge-on coplanar fits, which must be taken into account when testing for significance. Following the $\Delta \chi^{2}$ approach, we assume that $i_{p}, i_{B}$, and $\Delta \Omega$ are systematically adjusted, while the rest of the orbital parameters are fitted by our $N$-body model. The $\Delta \chi^{2}$ confidence intervals in this case obey the $\chi^{2}$ distribution for 3 degrees of freedom. The difference between the fits is $\Delta \chi^{2}=\chi_{\mathrm{CR}}^{2}-\chi_{\mathrm{MI}}^{2}=20.1$, suggesting that the best coplanar retrograde fit is between $3 \sigma$ and $4 \sigma$ worse than the mutually inclined fit, and thus the latter represents a significant model improvement.

Since the coplanar model with $p_{1}=11$ fitting parameters is "nested" within the mutually inclined model with $p_{2}=14$ parameters, another way to test the significance is the use of the $F$-test and determining the $F$-value following (Bevington \& Robinson 2003)

$$
F=\frac{\left(\chi_{\mathrm{CR}}^{2}-\chi^{2}\right) / \zeta_{1}}{\chi^{2} / \zeta_{2}}=\frac{\Delta \chi^{2} / \zeta_{1}}{\chi_{\nu}^{2}}=8.95
$$

where $\zeta_{1}=p_{2}-p_{1}$ is the number of additional parameters being tested and $\zeta_{2}=n-p_{2}$ is the number of degrees of freedom for the best mutually inclined model, with $n$ the number of data points. For $F=8.95$, the probability for model 
improvement is $p=0.000039$, which is much lower than our adopted cutoff value of $\alpha=0.01$, meaning that the null hypothesis is successfully rejected. Thus, we conclude that the mutually inclined fit is indeed better when compared to the coplanar edge-on model.

This significant model improvement is intriguing and deserves a closer look. First, it should be noted that the $\Delta \chi^{2}$ and $F$-tests only work well for Gaussian errors and models that are linear in the parameters (or could be linearized in the uncertainty region of the parameters due to large enough sample size; Press et al. 1992), which we do not have when we apply an $N$-body dynamical fit to the existing $\mathrm{RV}$ data for HD 59686.

Dynamical fitting of RV data consistent with two or more companions can be, in principle, sensitive to the true companion masses, but this has been proven to be very challenging even for the most extensively studied multiplanet systems (see Bean \& Seifahrt 2009; Correia et al. 2010; Nelson et al. 2016). The critical requirements to measure successfully mutual inclinations are (1) high RV precision; (2) low velocity jitter, typically on the order of at most a few $\mathrm{m} \mathrm{s}^{-1}$; (3) a large set of RV data covering many orbital cycles; and (4) a signal discrepancy between the minimum mass coplanar fit and the mutually inclined fit that is larger than the RV noise. In this context, we note that the available Lick data for HD 59686 do not satisfy these criteria, with only $88 \mathrm{RVs}$ (with precision of $5-9 \mathrm{~m} \mathrm{~s}^{-1}$ ) distributed over 11 yr covering only $\sim 40 \%$ of the outer binary orbit and RV jitter of $\sim 20 \mathrm{~m} \mathrm{~s}^{-1}$. Thus, it is unlikely that we would be able to tightly constrain the true companion masses (through $\Delta i, i_{p}$, and $i_{B}$ ).

In the bottom panel of Figure 1 , the RV residuals to the coplanar prograde fit are shown, and overplotted (red curve) is the difference of the mutually inclined model from the coplanar prograde one. Clearly, some of the outliers present in the coplanar prograde case are well modeled by the mutually inclined model with three additional fitting parameters. These outliers, however, lie in the relatively sparsely sampled epochs between JD $=2,454,200$ and 2,455,200, which unfortunately coincides with the outer binary periastron passage, when the RV signal changes rapidly. Perhaps the signal would be validated if we had more RV data following the mutually inclined fit prediction at that orbital phase, but currently, it is fair to conclude that we could be fitting noise rather than a true signal.

In addition, this mutually inclined best fit is extremely unstable. The inner companion has a nearly circular and highly inclined (polar-like) orbit with respect to the outer binary plane. Such orbits are potentially unstable due to the Lidov-Kozai effect (Kozai 1962; Lidov 1962), which leads to periodic exchanges between $\Delta i$ and $e_{p}$. Following the analytic expression given in Takeda \& Rasio (2005), we can estimate the inner companion maximum eccentricity $e_{p \text {, max }}$ that can be reached through the Lidov-Kozai cycle:

$$
e_{p, \max }=\sqrt{1-(5 / 3) \cos ^{2}(\Delta i)} .
$$

With $\Delta i=92.7$ obtained from the best fit, $e_{p, \max } \approx 0.998$, which exceeds our stability criterion. As expected, our direct numerical integration (right panel of Figure 3) shows that the best mutually inclined fit does not survive even $600 \mathrm{yr}$. The inner companion is quickly excited to $e_{p}>0.95$, and it eventually collides with the $\mathrm{K}$ giant. Obviously, such a highly inclined stellar triple is very unstable, which seems to be a good argument against this solution.

\section{Bootstrap Statistics}

Our goal in this section is to obtain parameter estimates and confidence regions based on the empirical distribution of constructed orbital parameters using bootstrap resampling. We analyze the distribution of the adjustable fitting parameters around the best fit by randomly drawing RV data points with replacement (e.g., Efron 1979; Press et al. 1992; Tan et al. 2013) and perform a dynamical fit to each RV data set obtained in this way.

We create a total of $n=5000$ bootstrapped data sets, and we fit each sample with strictly coplanar edge-on (prograde and retrograde) and mutually inclined configurations. All fits to the bootstrapped data sets are integrated for a maximum of $10 \mathrm{Myr}$, and their stability is examined. The $1 \sigma$ confidence levels from the distributions are used to estimate the asymmetrical best-fit parameter uncertainties. These estimates are listed in Table 1, along with the best-fit covariance matrix errors. For the mutually inclined fits, a bootstrapped sample is rejected if the fit suggests $i_{B}<30^{\circ}$ or $i_{B}>150^{\circ}$ (i.e., $\sin i_{B}<0.5$ ). This ensures that our samples are consistent with a reasonable secondary companion mass in the range $m_{B} \approx 0.53$ to about $1.1 M_{\odot}$ and thus are consistent with the observational constraints given in Ortiz et al. (2016). The total fraction of fits with highly inclined ( $\sin i_{B}<0.5$ ) outer companions is $\sim 6 \%$ of the total constructed bootstrapped RV data sets. In addition, $\sim 1.5 \%$ of the fits are unable to converge when we try to fit a mutually inclined configuration, meaning that in order to get 5000 fits, we have to create a total of $\sim 5400$ bootstrapped RV data sets.

Figure 5 shows the results from the bootstrap analysis for the edge-on $\left(i=90^{\circ}\right)$ prograde $\left(\Delta i=0^{\circ}\right)$ and retrograde $\left(\Delta i=180^{\circ}\right)$ configurations. In each panel, we illustrate the distribution of planet-versus-binary orbital elements $(K, P, e, \omega$, $M_{0}$ ), semimajor axes $a$, and dynamical masses $m$. Solid contours show the $1 \sigma, 2 \sigma$, and $3 \sigma(68.27 \%, 95.45 \%$, and $99.73 \%$ ) confidence levels from the two-dimensional parameter distributions. In all panels, the green dot represents the position of the best-fit values from the prograde or retrograde dynamical fit to the original data set, while the green error bars are the estimated uncertainties from the covariance matrix (see Table 1). With blue dots, we mark the unstable configurations, while with red points, we show the configurations that survive for $10 \mathrm{Myr}$.

Clearly, the distributions of orbital elements for the prograde and retrograde configurations are very similar. For both configurations, the covariance matrix errors estimated from the original data and the $1 \sigma(68.3 \%)$ confidence region from the bootstrap analysis are roughly consistent with each other. The main difference between the configurations comes from the stability results. We find that $\sim 97 \%$ of the retrograde configurations are stable for $10 \mathrm{Myr}$, while for the prograde case, this number is only $\sim 4 \%$. We note, however, that the stable prograde configurations fall mostly within the $1 \sigma$ confidence region, and from their distribution we can identify potentially stable regions of the parameter space. As can be seen in Figure 5, the stable fits seem to cluster around $\omega_{p} \approx \omega_{B} \approx 145^{\circ}, M_{0, B} \approx 257^{\circ}$ and $259^{\circ}, e_{p} \approx 0.05-0.08$, and a few discrete values in $P_{B}$ that avoid initial integer period ratios of 39 and 40 . This result implies that a small but robust 


\section{Prograde Coplanar Configuration}
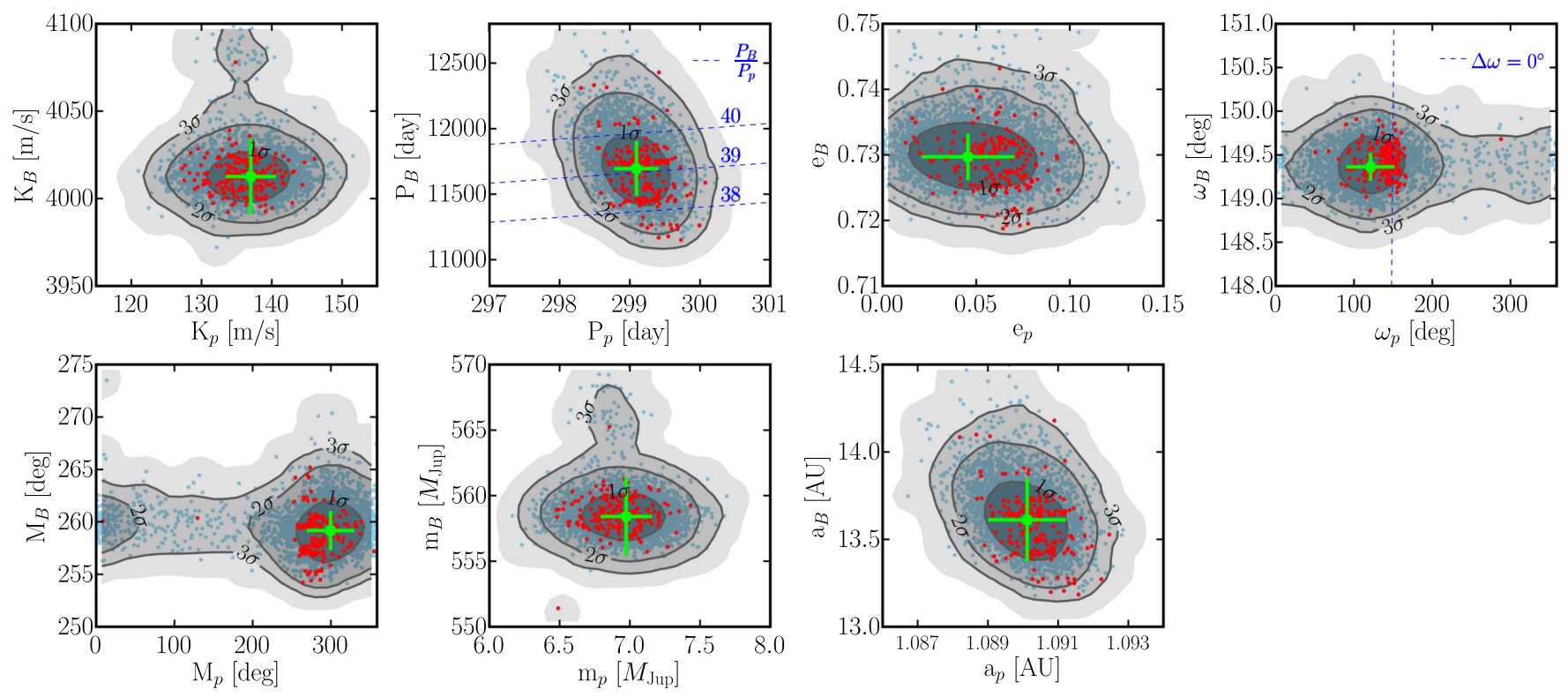

\section{Retrograde Coplanar Configuration}
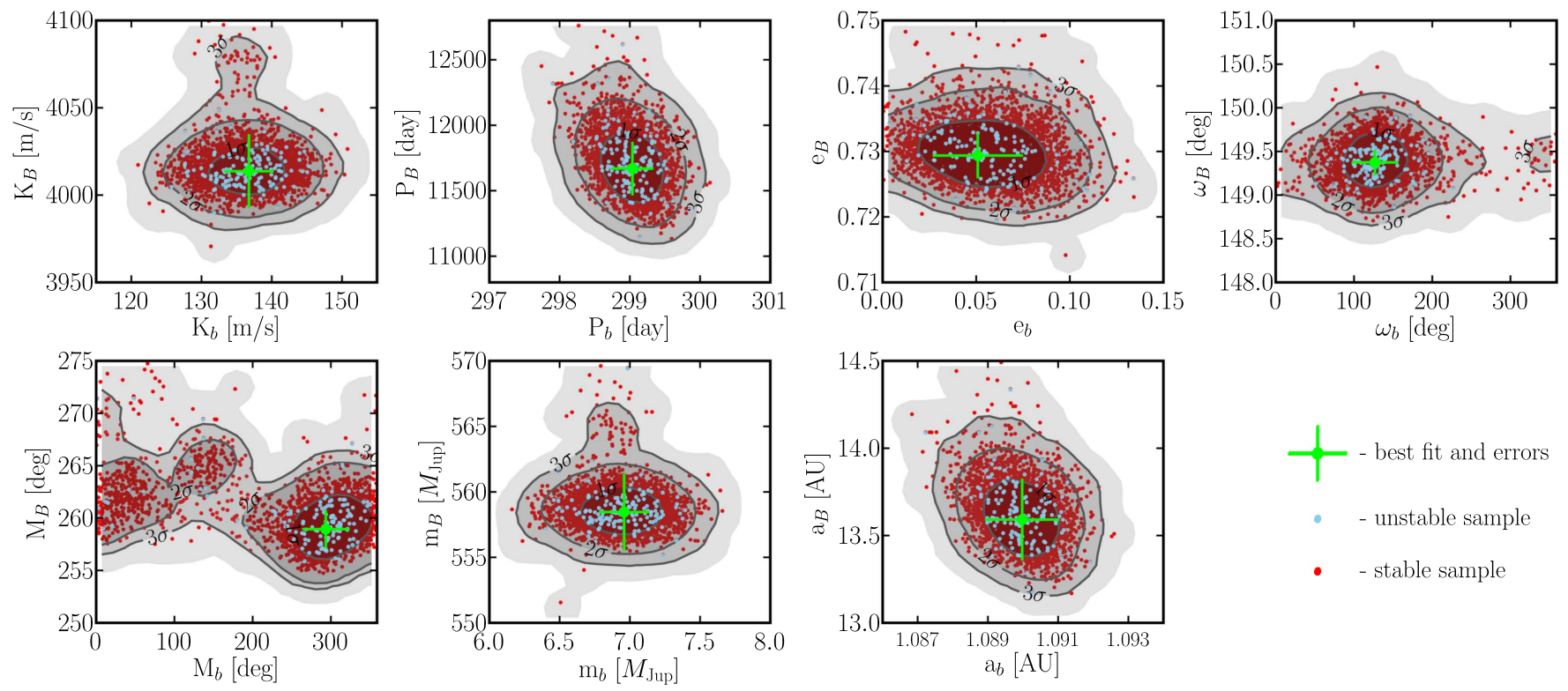

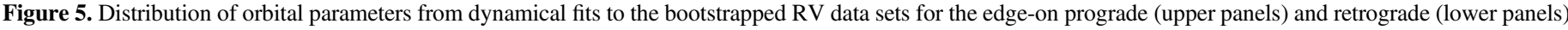

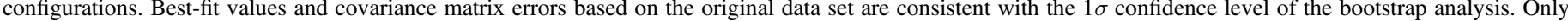

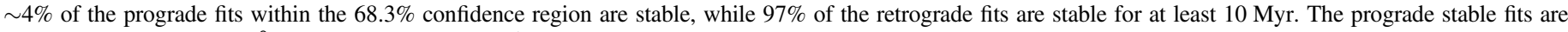
located at initial $\omega_{p} \approx 145^{\circ}, e_{p} \approx 0.05-0.08$, and $P_{B} / P_{p} \neq 39$ or 40 .

set of stable prograde fits does exist. Therefore, we cannot eliminate the possibility that the HD 59686 system is in a stable prograde configuration.

The results of the bootstrap analysis for the mutually inclined configuration are shown in Figure 6. In particular, we aim to quantitatively estimate the inclination distribution $\left(i_{p}, i_{B}, \Delta i\right)$ and see how often polar-like S-type companion orbits will occur in the resampled data. Therefore, in Figure 6, we introduce three additional panels: $\sin i_{p}-\sin i_{B}, \Delta i-\Delta \Omega$, and a comparison between the mutually inclined $\chi_{\text {mut }}^{2}$ and prograde coplanar $\chi_{\text {copl }}^{2}$ values. The last of these panels also shows the $F$-test probability for significant improvement when three additional parameters are added. We find that $\sim 86.1 \%$ of the fits lead to significant improvement, applying our chosen threshold of $\alpha=0.01$.

The orbital parameter distributions are wider than those in the coplanar cases but seem to agree with the best-fit errors. The binary orbit covariance matrix errors are sometimes larger than the bootstrap $1 \sigma$ contours, but in general, the distribution peak is consistent with the best-fit estimate. The inner companion inclination $i_{p}$ is found mostly at lower values, driving $m_{p}$ toward brown dwarf and starlike masses. On the other hand, within $3 \sigma, i_{B}$ stays between $75^{\circ}$ and $105^{\circ}$ (i.e., $\sin i_{B}$ between 1 and 0.96), leading to a value of $m_{B}$ similar to those of the edge-on cases. The distribution of $\Delta i$ clusters around the best fit and is well constrained around $\sim 90^{\circ}$, leading to nearly 


\section{Mutually Inclined Configuration}
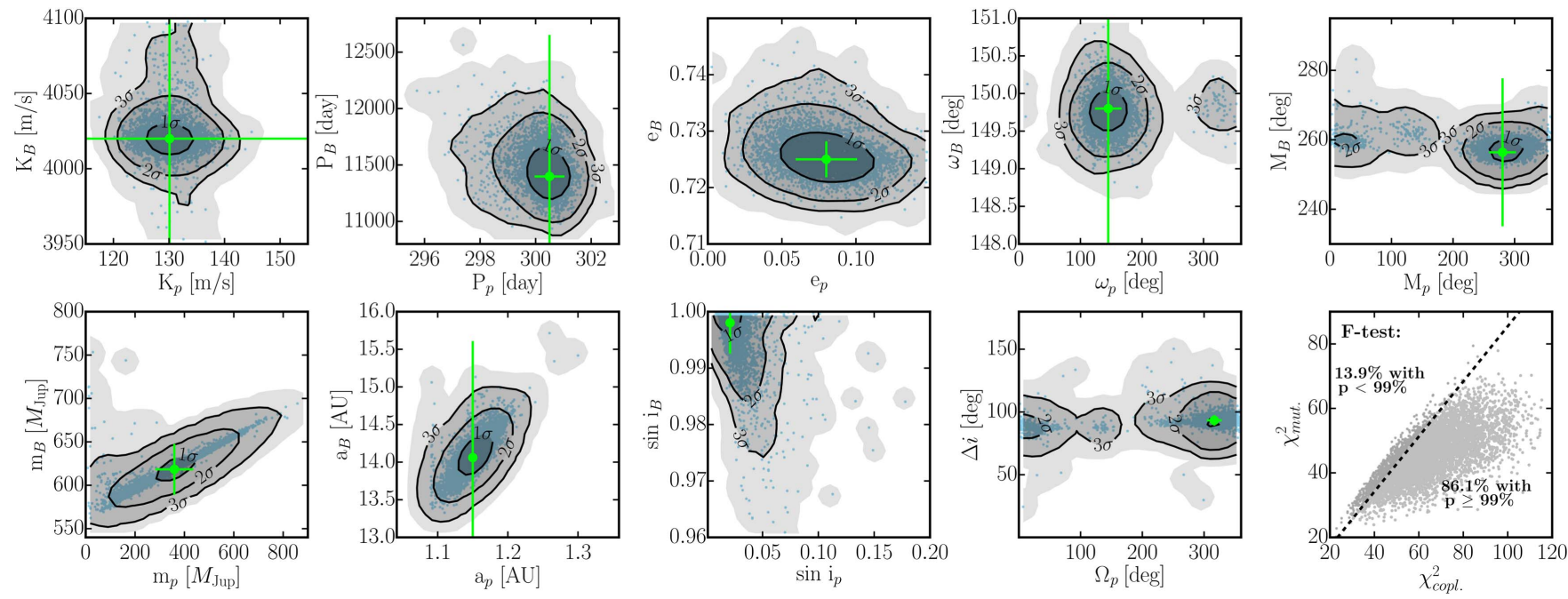

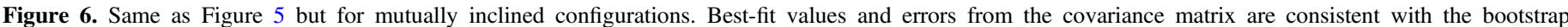

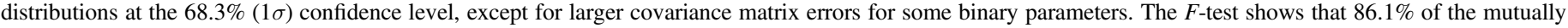
inclined fits present a significant improvement over the prograde fits, but none of them are stable.

perpendicular triple-star orbits. We suspect, however, that the distribution in $\Delta i$ might be the result of a model degeneracy. By randomly scrambling the data with repetition, the possibly problematic outliers with sparse cadence are not always removed (see Figure 1 and discussion in Section 4.3). Even worse, for some bootstrapped data sets, we repeat these points while removing data points with lower residuals. We find that none of the mutually inclined fits based on bootstrap analysis and shown in Figure 6 are long-term stable. The average bootstrap survival time is only a few hundred yr before the inner binary pair collides, which is consistent with the orbital evolution of the best mutually inclined fit to the original data.

\section{Parameter Grid Search}

A detailed picture of the dynamical properties of the HD 59686 system can also be assessed using the parameter grid search technique (e.g., Lee et al. 2006). We systematically vary a pair of orbital parameters, which we then keep fixed while the rest of the parameters in the model are adjusted to minimize $\chi^{2}$. This method allows us to systematically inspect the multidimensional parameter space around the best fit and study the properties and long-term stability of nearby fits.

\subsection{Coplanar Prograde Grids}

Our edge-on prograde bootstrap analysis reveals that stable fits are clustered around initial $\omega_{p} \approx 145^{\circ}, e_{p}=0.05-0.08$, and $P_{B} / P_{p} \neq 39$ or 40 (within $1 \sigma$ from the best fit). These three orbital parameters are also the least-constrained parameters from our fitting, especially when compared to $P_{p}, \omega_{B}$, and $e_{B}$. Therefore, any grid combination including $P_{B}, \omega_{p}$, and $e_{p}$ yields an adequate search for prograde coplanar stable fits. Figure 7 shows stability results for an edge-on coplanar grid in the $P_{B} / P_{p}-e_{p}$ space, where we fix $P_{p}$ and $\omega_{B}$ at their best-fit values of 299.1 days and $149^{\circ} .4$, respectively. Since we know from the bootstrap analysis that stability appears when $\Delta \omega \approx 0^{\circ}$, we keep $\omega_{p}=\omega_{B}$ fixed, leading to an initially aligned configuration with $\Delta \omega=0^{\circ}$. In Figure 7, stability within the $1 \sigma$ confidence region from the best fit is achieved

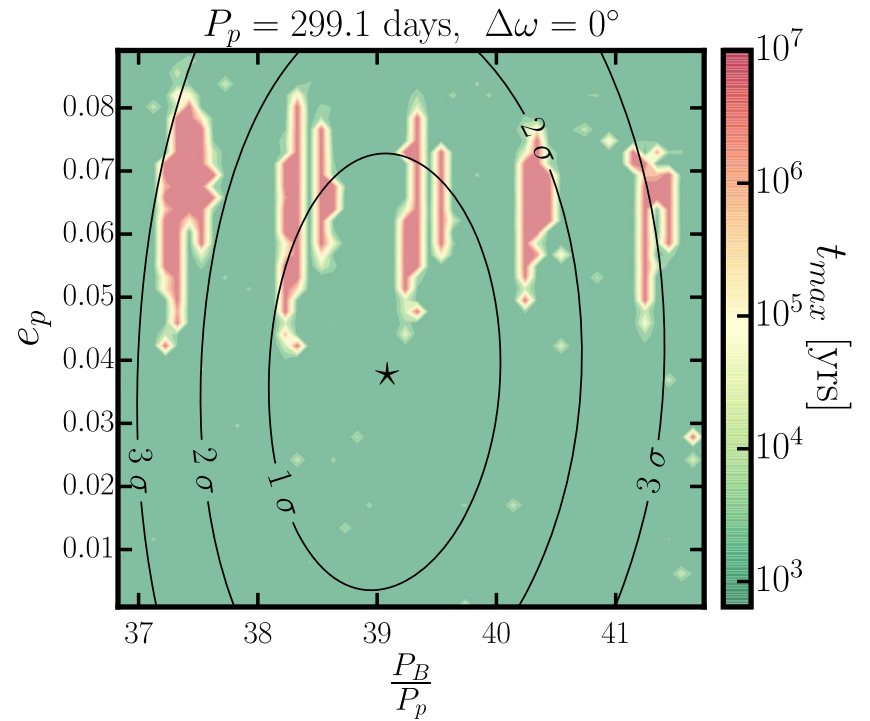

Figure 7. Edge-on coplanar prograde grid in the $P_{B} / P_{p}-e_{p}$ space, where $P_{p}$ and $\omega_{B}$ are fixed at their best-fit values of 299.1 days and $149^{\circ} .4$, respectively, while $\omega_{p}$ is also fixed to $149^{\circ} .4$ to assure $\Delta \omega=0^{\circ}$. The time for which the system is stable is color-coded. Black contours are the confidence levels corresponding to the $1 \sigma, 2 \sigma$, and $3 \sigma$ confidence regions of the $\chi_{\nu}^{2}$ minimum (black star). For this aligned grid, stability is achieved only in the range of initial $e_{p} \approx 0.05-0.08$ and $P_{B} / P_{p} \neq \mathbb{Z}$.

when $e_{p} \approx 0.05-0.08$ and $P_{B} / P_{p} \neq 39$ or 40 , which confirms the results from the bootstrap analysis.

As a next step, we test for stability in eight $P_{B}-\omega_{p}$ grids, each with $e_{p}=0.02-0.09$ in steps of 0.01 . Here $P_{B}$ and $\omega_{p}$ are varied around the best coplanar fit in the ranges of $11,000-12,500$ days and $60^{\circ}-210^{\circ}$, respectively, while the remaining parameters in the dynamical model are adjusted. Figure 8 shows the survival time resulting from this test, together with the confidence levels. Since these grids are constructed with three systematically varied parameters $\left(P_{B}\right.$, $\omega_{p}$, and $e_{p}$ ), we consider Figure 8 as a three-dimensional parameter cube, where each grid is a separate $P_{B}-\omega_{p}$ slice placed on a lower-resolution " $z$ "-axis constructed for different 

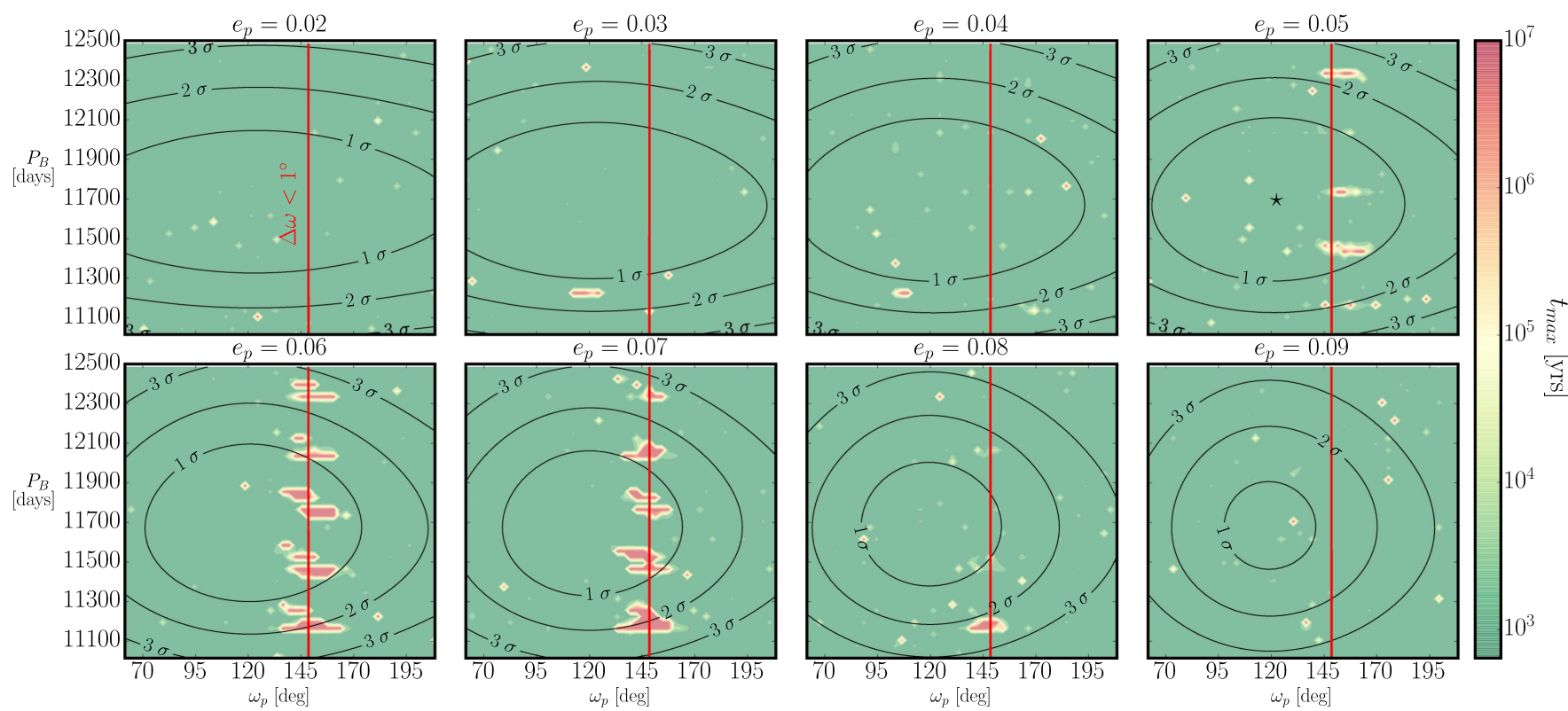

Figure 8. Edge-on coplanar prograde grids for systematically varied $P_{B}, \omega_{p}$, and $e_{p}$ around their best-fit values. The survival time is color-coded, and black contours correspond to the confidence levels of the best fit. Most of the fits are highly unstable, except those located between an integer period ratio and near alignment $\left(\Delta \omega \approx 0^{\circ}\right)$, while $e_{p}$ is between 0.05 and 0.07 . Some of these stable islands are within the $1 \sigma$ confidence level of the best fit and provide valid possibilities for the orbital configuration of the HD 59686 system.
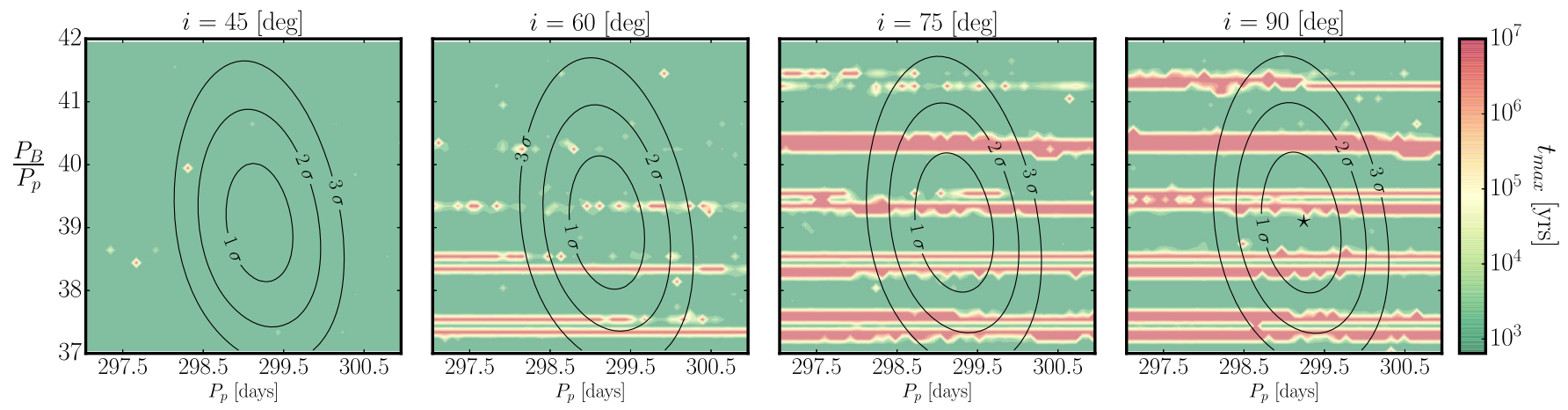

Figure 9. Coplanar prograde grids of $P_{B} / P_{p}$ vs. $P_{p}$ for fixed $e_{p}=0.06, \omega_{p}=\omega_{B}=149^{\circ} .4$ (i.e., $\Delta \omega=0^{\circ}$ ), and $i=90^{\circ}, 75^{\circ}, 60^{\circ}$, and $45^{\circ}$. The stable regions seen in Figure 7 are now extended through the whole range of $P_{p}$ and cross inside the $1 \sigma$ confidence levels of the best fit. These stable regions exist down to $i=60^{\circ}$, below which both companions have masses large enough to make the system unstable.

$e_{p}$. Thus, the significance levels (black contours) shown in Figure 8 are calculated for three degrees of freedom. Clearly, most of the fits are highly unstable, except those located between integer period ratios with nearly aligned orbits $\left(|\Delta \omega| \lesssim 10^{\circ}\right)$ and $e_{p}$ between 0.05 and 0.07 . We find that these stable islands cover the largest area when $e_{p}=0.06$.

Knowing that $e_{p}$ and $\omega_{p}$ are critical stability parameters, in Figure 9 , we show results for $P_{B} / P_{p}-P_{p}$ grids, where we fix $e_{p}=0.06$ and $\omega_{p}=\omega_{B}=149^{\circ} .4$, and we systematically adopt inclinations of $i=90^{\circ}, 75^{\circ}, 60^{\circ}, 45^{\circ}$, and $30^{\circ}$. In this way, we study the stability of fits in the $P_{B} / P_{p}-P_{p}$ parameter space for initially aligned orbits and increasing companion masses. In these grids, the stable regions now extend through a large range of $P_{p}$ and cross inside the $1 \sigma$ confidence regions. Configurations with period ratios near an integer initially are highly unstable, while stable configurations can be found between initial integer period ratios. The stable regions are evident in the $i=90^{\circ}-60^{\circ}$ grids, but they become smaller with decreasing $i$ or increasing masses. The $i=45^{\circ}$ grid contains only a few marginally stable fits, which are likely unstable beyond $10 \mathrm{Myr}$, while all configurations for the $i=30^{\circ}$ grid (not shown in
Figure 9) are unstable. These results identify a lower limit for the inclination of $i \approx 45^{\circ}$ for stable prograde coplanar configurations. This inclination limit happens to coincide with the secondary-star mass constraints discussed in Ortiz et al. (2016).

Table 2 gives the orbital parameters and corresponding errors for the best stable fit among these grids, which has an initial $P_{B} / P_{p} \approx 39.3$. Figure 10 shows the orbital evolution of this stable fit. The evolution of the semimajor axes $a_{p}$ and $a_{B}$ in the upper left panel shows that this configuration remains longterm stable with well-separated orbits. The binary eccentricity $e_{B}$ has very small amplitude variations around 0.73 , while the planet remains nearly circular, with $e_{p}$ varying between 0.04 and 0.11 (upper right panel). The bottom two panels of Figure 10 show the evolution of the secular apsidal angle $\Delta \omega=\omega_{\mathrm{p}}-\omega_{\mathrm{B}}$, which exhibits a clear libration around $0^{\circ}$ with a semi-amplitude of $\pm 37^{\circ}$, while the mean period ratio during the integration is $\approx 39.4$, close to the initial $P_{B} / P_{p}$. We examine this configuration for librating resonance angles (see Equation (3)) associated with the nearest 39:1 and 40:1 mean-motion commensurabilities and confirm that this stable 
Table 2

Stable Prograde Fit with $P_{B} / P_{p} \approx 39.3$

\begin{tabular}{lcc}
\hline \hline Parameter & HD $59686 \mathrm{Ab}$ & HD 59686 B \\
\hline$K\left[\mathrm{~m} \mathrm{~s}^{-1}\right]$ & $137.0 \pm 3.3$ & $4011.6 \pm 3.7$ \\
$P[$ days] & $299.2^{\mathrm{a}}$ & $11772.7^{\mathrm{a}}$ \\
$e$ & $0.06^{\mathrm{a}}$ & $0.731 \pm 0.005$ \\
$\omega[\mathrm{deg}]$ & $149.3^{\mathrm{a}}$ & $149.4 \pm 0.1$ \\
$M_{0}[\mathrm{deg}]$ & $272.0 \pm 1.4$ & $259.8 \pm 0.4$ \\
$\mathrm{RV}_{\mathrm{off}}\left[\mathrm{m} \mathrm{s}^{-1}\right]$ & $243.2 \pm 4.0$ & $\ldots$ \\
$i[\mathrm{deg}]$ & $90.0^{\mathrm{a}}$ & $90.0^{\mathrm{a}}$ \\
$\Omega[\mathrm{deg}]$ & $0.0^{\mathrm{a}}$ & $0.0^{\mathrm{a}}$ \\
$\Delta i[\mathrm{deg}]$ & 0.0 & $\ldots$ \\
$a[\mathrm{au}]$ & 1.09 & 13.67 \\
$m$ sin $i\left[M_{\mathrm{Jup}}\right]$ & 6.97 & 558.5 \\
$\mathrm{rms}\left[\mathrm{m} \mathrm{s}^{-1}\right]$ & 19.84 & $\ldots$ \\
$\chi^{2}$ & 78.57 & $\ldots$ \\
$\chi_{\nu}^{2}$ & 0.970 & $\ldots$ \\
\hline
\end{tabular}

${ }^{\mathrm{a}}$ Fixed parameters.

prograde fit is not involved in an MMR. Such an orbital evolution is characteristic for secular apsidal alignment (e.g., Lee \& Peale 2003; Michtchenko \& Malhotra 2004). The libration of $\Delta \omega$ around $0^{\circ}$ is critical for the stability of our system, since it helps the lower-mass S-type object to retain small eccentricities while being significantly perturbed by the secondary star. We have investigated all stable prograde islands shown in Figures 7-9, and they all exhibit similar evolution, with librating $\Delta \omega$ around $0^{\circ}$, circulating MMR angles $\theta_{1, n}$, small $e_{p}$, and a noninteger mean period ratio $P_{B} / P_{p}$. Thus, we conclude that if the HD 59686 system is indeed prograde, then it must be locked in secular apsidal alignment to stabilize the orbits.

Two additional remarks on the secular apsidal alignment and non-MMR nature of the stable islands in Figures 7-9 are in order. First, one may be concerned that the noninteger initial and mean period ratios may not represent the true period ratio due to the large mass of the secondary star. However, the Hamiltonian in Jacobi coordinates in Equation (11) of Lee \& Peale (2003) shows that the perturbations to the Keplerian motions from the interactions between the secondary star and the planet remain small, and the semimajor axes and period ratio should be nearly constant throughout most of the binary orbit (including the initial epoch when the secondary is $\sim 20$ au from the primary). Even when the secondary is at periastron, we can estimate from the lowest-order term in the perturbations to the Kepler motions in Equation (11) of Lee \& Peale (2003) that the full amplitude of the variation in the period ratio should be $\sim(9 / 2)\left(m_{B} / M_{*}\right)\left(a_{p} / a_{B}\left(1-e_{B}\right)\right)^{3} \sim 3.3 \%$ if $e_{p}$ is small. These results are consistent with the evolution of $P_{B} / P_{p}$ shown in Figure 10, where $P_{B} / P_{p}$ is near the initial value most of the time and shows scatter of $\sim 4.5 \%$.

Second, K. H. Wong \& M. H. Lee (2018, in preparation) systematically studied the stability of circumprimary planetary orbits in the HD 59686 system, with initial conditions in grids of $a_{p}$ and $e_{p}$ for several values of $\Delta \omega$ and mean anomalies. For the coplanar prograde case, they confirmed the existence of islands stabilized by secular apsidal alignment. They also found islands that are stabilized by MMR, but these are at higher $e_{p}$ and do not fit the observed planet.

\subsection{Coplanar Retrograde Grids}

We repeat the grid analysis for retrograde coplanar configurations by fixing $\Delta i=180^{\circ}$ for each fit. We find that all fits within $3 \sigma$ from the best fit are stable for at least $10 \mathrm{Myr}$. Particularly, all fits in the $P_{B} / P_{p}-P_{p}$ grids are stable despite the large companion masses for $i=30^{\circ}$ and even $i=15^{\circ}$. The stability of the $P_{B} / P_{p}-P_{p}$ grids in the range $i=90^{\circ}-15^{\circ}$ is in agreement with the results presented in Figure 4 and Section 4.2, where all the retrograde coplanar inclined best fits are stable down to $i=5^{\circ}$. We conclude that the best retrograde coplanar fit is well within a large stable phase-space region, not necessarily involved in an MMR. Therefore, no meaningful stability constraints can be obtained from the retrograde coplanar grids, except that the retrograde orbits yield very strong candidates for the HD 59686 system configuration.

\subsection{Mutually Inclined Grids}

We construct 12 separate $i_{B}-i_{p}$ grids, where for each grid we adopt $\Delta \Omega=0^{\circ}-330^{\circ}$ with a step size of $30^{\circ}$. In these grids, $i_{B}$ and $i_{p}$ are varied from $5^{\circ}$ to $175^{\circ}$ with a step size of $3^{\circ} .4$, corresponding to 50 different values. Fits with $i_{p}$ and $i_{B}$ below $5^{\circ}$ and above $175^{\circ}$ are not considered, since these inclinations lead to nearly face-on orbits, and the dynamical masses of the companions will be very large. In fact, as discussed in Section 4.2 , other observations constrain $\sin i_{B}$ to values greater than 0.5 , but since the dynamical model does not reject a more massive secondary stellar companion, we construct symmetrical grids with the same range of $i_{p}$ and $i_{B}$. Thus, we study mutually inclined configurations covering almost all possible system geometries. The results from this test are shown in Figure 11.

The only stable region we find in these grids corresponds to nearly coplanar retrograde geometries with $\Delta i>145^{\circ}$. The orbital evolution of these stable fits is very similar to that of the best retrograde coplanar fit discussed in Section 4.1 and shown in the middle panel of Figure 3. For nearly coplanar retrograde orbits, the amplitude of the variations in the inner companion eccentricity $e_{p}$ is rather large. It is about 0.35 for strictly coplanar orbits and increases with the increasing mass of the secondary star (i.e., with decreasing $\sin i_{B}$ ) to about 0.42 at maximum, when $i_{B}$ and $\Delta i \approx 145^{\circ}$. Meanwhile, the $e_{p}$ and $e_{B}$ oscillation frequency is highest in the coplanar configuration and decreases with decreasing $\Delta i$. For fits near the stability boundary of $\Delta i \approx 145^{\circ}$, another shorter-term eccentricity variation is visible on top of the main secular eccentricity oscillation, which has a period of a few hundred yr. In this stable region, $\Delta i$ also exhibits small variations around the initial best-fit value, except for the coplanar case, where $\Delta i$ remains constant at $180^{\circ}$. We do not find evidence that any of the stable retrograde fits are in MMR.

The small $\sin i_{p}$ retrograde corners of Figure 11 are intriguingly stable, suggesting that the inner companion's dynamical mass could be as high as 10 times its minimum mass (i.e., $\sin i_{p} \approx 0.1$ ), converting the planet to a massive brown dwarf or, at the extreme, even a very low-mass M-dwarf star. A massive, highly inclined, and retrograde inner companion may be consistent with the Hipparcos astrometry and dynamical modeling, but to preserve the system's stability, the outer binary companion must also have a small $\sin i_{B}$, and that is not supported by observations. On the one hand, if we assume that the outer companion is a main-sequence star, then we can limit 

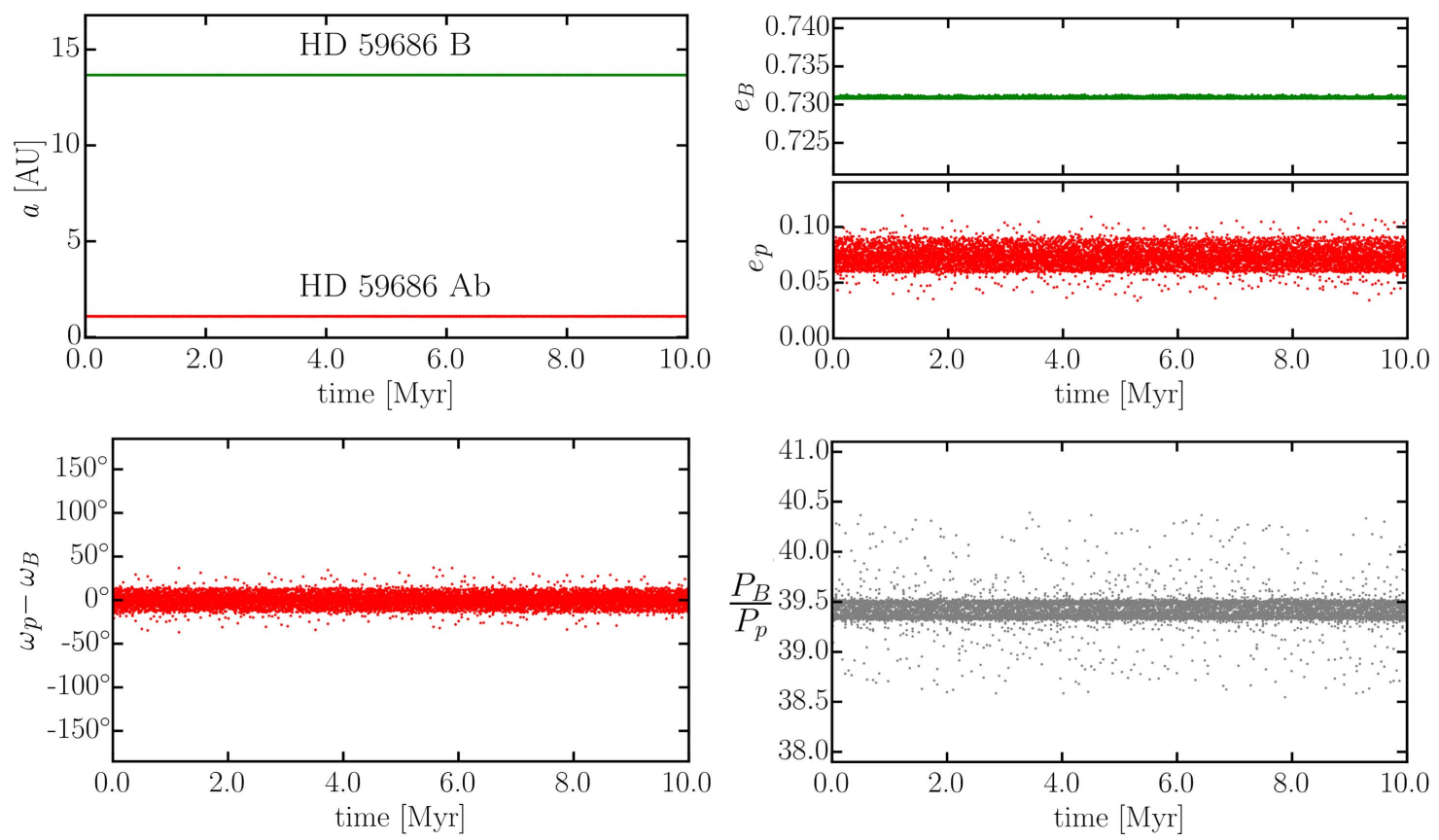

Figure 10. Top panels: evolution of the binary (green) and planetary (red) semimajor axes and eccentricities of the best stable coplanar, edge-on, and prograde fits with initial $P_{B} / P_{p} \approx 39.3$. No notable changes can be seen in $a_{p}$ and $a_{B}$. The binary eccentricity $e_{B}$ fluctuates with a very small amplitude around 0.73 , while $e_{p}$ librates with a larger amplitude between 0.04 and 0.11 . Bottom panels: This fit is clearly locked in secular apsidal alignment, where the secular apsidal angle $\Delta \omega=\omega_{p}-\omega_{B}$ librates around $0^{\circ}$ with a semi-amplitude of $\pm 37^{\circ}$, while the mean orbital period ratio is $P_{B} / P_{p} \approx 39.4$.

its inclination to $150^{\circ} \geqslant i_{B} \geqslant 30^{\circ}$ (blue dashed lines in Figure 11), beyond which it would be a solar-mass star and should have been detected in Ortiz et al. (2016). All stable solutions with secondary $150^{\circ} \geqslant i_{B} \geqslant 30^{\circ}$ support a planetmass object for the inner companion. On the other hand, the LBT observations obtained in Ortiz et al. (2016) would not be sensitive to a white dwarf secondary with $\sim 1 M_{\odot}$. Thus, in principle, both companions of HD 59686 can have very small $\sin i$, making the system a hierarchical retrograde triple of a $\mathrm{K}$ giant, M dwarf, and white dwarf. Such an exotic system would be stable in a retrograde orbit, but then, in all cases, the excited inner binary eccentricity would oscillate with much larger values than the one currently observed; i.e., our observations would have caught the system at a very special time. Therefore, we conclude that the inner companion is most likely of planetary origin.

Overall, we identify a large and confident stable region for the HD 59686 system, which turns out to be at high mutual inclinations of $\Delta i \gtrsim 145^{\circ}$, corresponding to nearly coplanar but retrograde orbits. Mutual inclinations with $\Delta i$ between $30^{\circ}$ and $145^{\circ}$ lead to instability on very short timescales due to Lidov-Kozai effects, and thus such orbital configurations are very unlikely. Nearly coplanar and prograde best fits with $\Delta i \lesssim 30^{\circ}$ are also unstable.

\section{Summary and Conclusions}

HD 59686 is without any doubt a very interesting three-body system. It consists of a single-lined spectroscopic binary with a K-giant primary with $M=1.92 M_{\odot}$, a low-mass secondary star with at least $m_{B}=0.53 M_{\odot}$ and at most $\sim 1 M_{\odot}$, and an additional S-type planet with at least $7 M_{\text {Jup }}$. This system has a challenging architecture, since the secondary star orbits beyond the planet orbit $\left(a_{p}=1.09 \mathrm{au}\right)$ on a relatively close $\left(a_{B}=13.6 \mathrm{au}\right)$ and very eccentric $\left(e_{B}=0.73\right)$ orbit. As a result, the binary periastron distance is only $q_{B}=3.7 \mathrm{au}$, leading to strong interactions with the planet and thus challenging the system's long-term stability.

In this paper, we performed a detailed orbital and stability analysis of the HD 59686 system via dynamical modeling of $\mathrm{RV}$ data and long-term $N$-body integrations. We aimed to refine the orbital parameters by stability constraints, which can provide clues on the formation history. This is important, since only a handful of S-type planetary candidates in compact binary systems are known in the literature, and the HD 59686 system illustrates how planets could form and remain stable in an S-type orbit around a star under the strong gravitational influence from a close stellar secondary.

Our global best fit with the lowest $\chi_{\nu}^{2}$ suggests a triple-star system with nearly polar orbits $\left(\Delta i=92^{\circ} .7 \pm 3^{\circ} .3\right)$ instead of a binary with an S-type planet. We have shown, however, that such orbits quickly lead to instability due to the Lidov-Kozai effect. Within only $600 \mathrm{yr}$, the Lidov-Kozai effect excites the eccentricity of the inner object to a value that leads to collision with the primary K-giant star. Orbital fits with parameters similar to those of the near-polar global best fit are very unstable and experience a similar fate, although they all have lower $\chi_{\nu}^{2}$ values compared to the fits corresponding to a coplanar configuration. We conclude that the near-polar configurations cannot represent the true system configuration, and that their small $\chi_{\nu}^{2}$ values are most likely a result of model degeneracies, which come from the limited number and accuracy of RV data points. These conclusions are supported by our bootstrap statistical analysis.

We find that HD 59686's planet can only survive on nearly coplanar, most likely retrograde orbits. We find that when the system's mutual inclination is $\Delta i=180^{\circ}$ (i.e., coplanar and retrograde), the system is fully stable for a large set of orbital solutions and companion masses. Long-term stability is also preserved for nearly coplanar retrograde configurations with $145^{\circ} \lesssim \Delta i \leqslant 180^{\circ}$. 

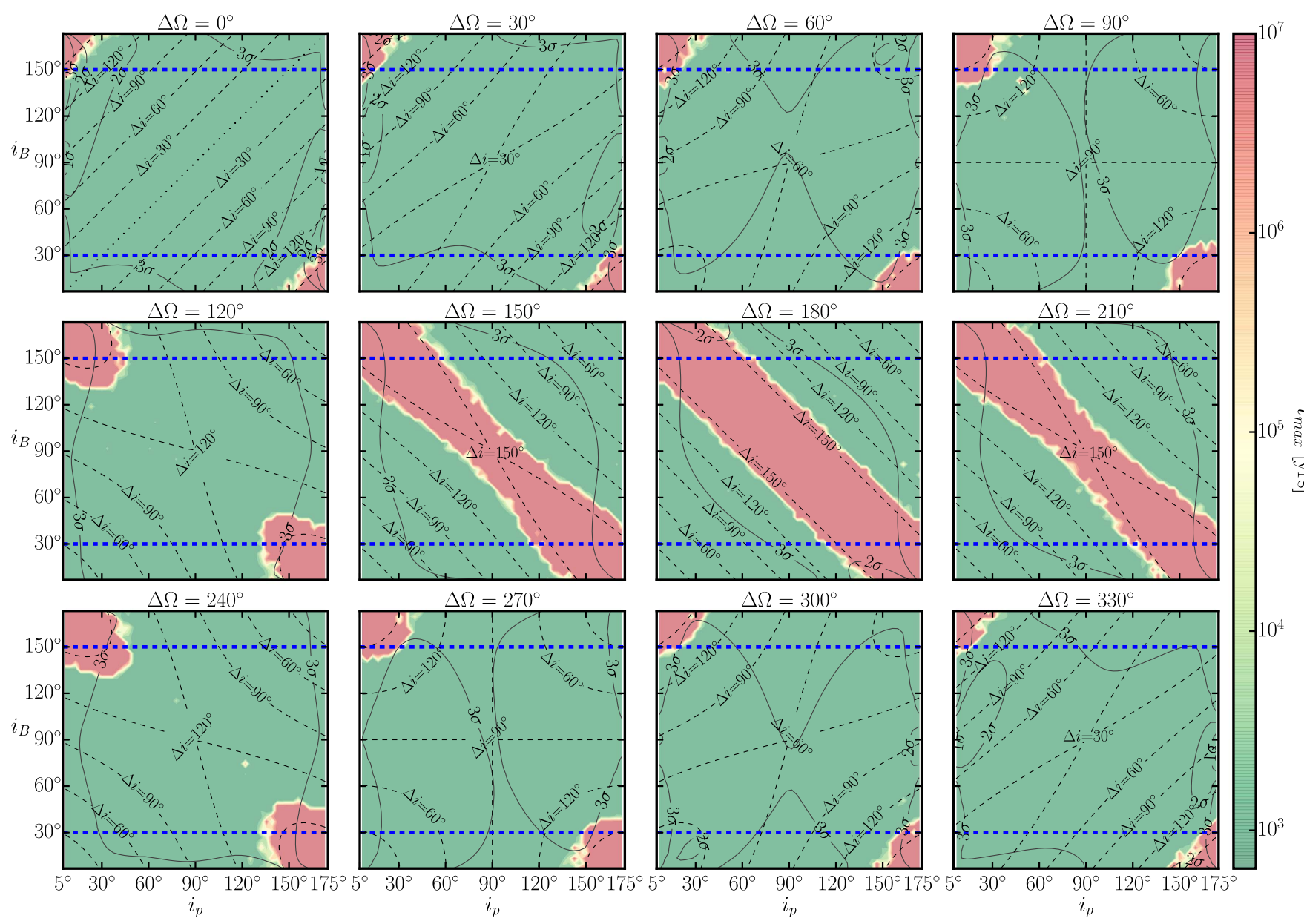

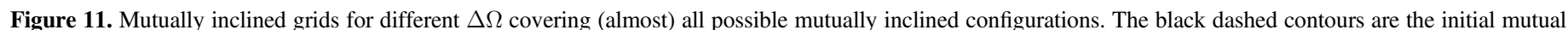

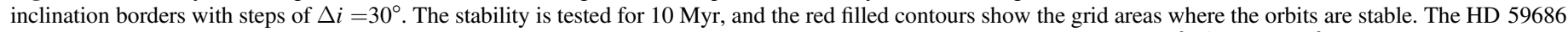

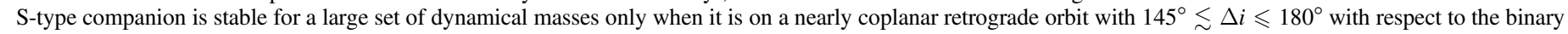
plane.

Although most of the coplanar prograde fits consistent with HD 59686's RV data are unstable, we have demonstrated that stable prograde fits do in fact exist. Our bootstrap and grid search analysis shows that a fraction of prograde fits (mostly within $1 \sigma$ from the best fit) are stable for at least $10 \mathrm{Myr}$. These fits are located in narrow strips of the orbital period space where the initial period ratio $P_{B} / P_{p}$ is not an integer number, with the best chances of stability near $P_{B} / P_{p} \approx 38.4$ and 39.4 . Therefore, the system can only survive between high-order MMRs, while the MMRs themselves have a destabilizing effect on the S-type planet. However, we find that the planetary $e_{p}$ and $\omega_{p}$ are also very important parameters that control the planet stability. The stability results indicate that the planet must be initially on a nearly aligned orbit with the binary $\left(\Delta \omega \leqslant 10^{\circ}\right)$ and have $e_{p} \approx 0.06$. In such a configuration, longterm stability is ensured by secular apsidal alignment between the binary and planetary orbits, with $\Delta \omega$ librating around $0^{\circ}$ with a relatively small amplitude.

The stable islands shrink when we assume lower inclinations and more massive companions while keeping coplanarity. Below $i=45^{\circ}$, all prograde coplanar configurations are unstable, which suggests that if the system is indeed prograde and coplanar, then $90^{\circ} \geqslant i \geqslant 45^{\circ}$, with most stable fits at $i=90^{\circ}$. The orbital dynamics of these stable prograde fits with larger masses similarly shows secular apsidal alignment where $\Delta \omega$ librates around $0^{\circ}$.

As a final discussion point, we note that there are arguments in favor of and against both prograde and retrograde configurations. For example, the retrograde stable region is very large, and it can explain the RV data with great confidence, but forming a retrograde planet requires some exotic scenarios (see discussion in Ortiz et al. 2016). On top of that, looking at the retrograde planet eccentricity evolution (middle panel of Figure 3), we estimate that $\sim 18 \%$ of the time, $e_{p}<0.1$ (within $3 \sigma$ of the best-fit value), and only $\sim 8 \%$ of the time, $0.03<e_{p}<0.07$ (within $1 \sigma$ ). If the system is indeed in a retrograde configuration, then the Lick RVs must have been obtained in a phase that has rather low probability when $e_{p}$ is as low as $\sim 0.05$. On the other hand, small $e_{p}$ is not a problem for the prograde stable fits, where most of the time the planet eccentricity is in the range $0.04<e_{p}<0.11$ (Figure 10). However, the stable prograde islands are very narrow, and whether it is possible to form a massive planet in a narrow stable region in secular apsidal alignment with the eccentric close binary is a problem that deserves a closer look in the future. 
TT and MHL are supported in part by Hong Kong RGC grant HKU 17305015. We thank the anonymous referee for the excellent comments that helped to improve this paper.

\section{ORCID iDs}

Trifon Trifonov (iD https://orcid.org/0000-0002-0236-775X

Man Hoi Lee (iD https://orcid.org/0000-0003-1930-5683

Sabine Reffert (ib) https://orcid.org/0000-0002-0460-8289

\section{References}

Baluev, R. V. 2009, MNRAS, 393, 969

Barban, C., De Ridder, J., Mazumdar, A., et al. 2004, in ESA Special Publication 559, SOHO 14 Helio- and Asteroseismology: Towards a Golden Future, ed. D. Danesy (San Francisco, CA: ASP), 113

Bean, J. L., \& Seifahrt, A. 2009, A\&A, 496, 249

Bevington, P. R., \& Robinson, D. K. 2003, Data Reduction and Error Analysis for the Physical Sciences (Boston, MA: McGraw-Hill)

Borucki, W. J., Koch, D., Basri, G., et al. 2010, Sci, 327, 977

Campbell, B., Walker, G. A. H., \& Yang, S. 1988, ApJ, 331, 902

Chauvin, G., Beust, H., Lagrange, A.-M., \& Eggenberger, A. 2011, A\&A, 528, A8

Correia, A. C. M., Couetdic, J., Laskar, J., et al. 2010, A\&A, 511, A21

Correia, A. C. M., Udry, S., Mayor, M., et al. 2008, A\&A, 479, 271

Doyle, L. R., Carter, J. A., Fabrycky, D. C., et al. 2011, Sci, 333, 1602

Dvorak, R. 1986, A\&A, 167, 379

Eberle, J., \& Cuntz, M. 2010, ApJL, 721, L168

Efron, B. 1979, AnSta, 7, 1

Eggenberger, A., \& Udry, S. 2010, in EAS Publications Ser., Vol. 41, ed. T. Montmerle, D. Ehrenreich, \& A.-M. Lagrange (Les Ulis: EDP Sciences), 27

Eggleton, P., \& Kiseleva, L. 1995, ApJ, 455, 640

Frink, S., Quirrenbach, A., Fischer, D., Röser, S., \& Schilbach, E. 2001, PASP, 113,173

Giuppone, C. A., Morais, M. H. M., Boué, G., \& Correia, A. C. M. 2012, A\&A, 541, A151

Goździewski, K., Słonina, M., Migaszewski, C., \& Rozenkiewicz, A. 2013, MNRAS, 430, 533

Haghighipour, N. 2006, ApJ, 644, 543

Hamilton, D. P., \& Burns, J. A. 1992, Icar, 96, 43

Hatzes, A. P., Cochran, W. D., Endl, M., et al. 2003, ApJ, 599, 1383

Hekker, S., \& Meléndez, J. 2007, A\&A, 475, 1003
Hekker, S., Reffert, S., Quirrenbach, A., et al. 2006, A\&A, 454, 943

Holman, M. J., \& Wiegert, P. A. 1999, AJ, 117, 621

Kaeufl, H.-U., Ballester, P., Biereichel, P., et al. 2004, Proc. SPIE, 5492, 1218

Kjeldsen, H., \& Bedding, T. R. 2011, A\&A, 529, L8

Kostov, V. B., McCullough, P. R., Hinse, T. C., et al. 2013, ApJ, 770, 52

Kozai, Y. 1962, AJ, 67, 591

Lee, M. H., Butler, R. P., Fischer, D. A., Marcy, G. W., \& Vogt, S. S. 2006, ApJ, 641, 1178

Lee, M. H., \& Peale, S. J. 2003, ApJ, 592, 1201

Leung, G. C. K., \& Lee, M. H. 2013, ApJ, 763, 107

Lidov, M. L. 1962, P\&SS, 9, 719

Mardling, R. A. 2008, in The Cambridge N-Body Lectures, ed. S. J. Aarseth, C. A. Tout, \& R. A. Mardling (Berlin: Springer), 59

Mardling, R. A., \& Aarseth, S. J. 2001, MNRAS, 321, 398

Michtchenko, T. A., \& Malhotra, R. 2004, Icar, 168, 237

Morais, M. H. M., \& Giuppone, C. A. 2012, MNRAS, 424, 52

Nelson, B. E., Robertson, P. M., Payne, M. J., et al. 2016, MNRAS, 455, 2484

Ortiz, M., Reffert, S., Trifonov, T., et al. 2016, A\&A, 595, A55

Press, W. H., Teukolsky, S. A., Vetterling, W. T., \& Flannery, B. P. 1992, Numerical Recipes in FORTRAN. The Art of Scientific Computing (Cambridge: Cambridge Univ. Press)

Ramm, D. J. 2015, MNRAS, 449, 4428

Ramm, D. J., Nelson, B. E., Endl, M., et al. 2016, MNRAS, 460, 3706

Ramm, D. J., Pourbaix, D., Hearnshaw, J. B., \& Komonjinda, S. 2009, MNRAS, 394, 1695

Reffert, S., Bergmann, C., Quirrenbach, A., Trifonov, T., \& Künstler, A. 2015, A\&A, 574, A116

Reffert, S., \& Quirrenbach, A. 2011, A\&A, 527, A140

Roell, T., Neuhäuser, R., Seifahrt, A., \& Mugrauer, M. 2012, A\&A, 542, A92

Takeda, G., \& Rasio, F. A. 2005, ApJ, 627, 1001

Tan, X., Payne, M. J., Lee, M. H., et al. 2013, ApJ, 777, 101

Thebault, P., \& Haghighipour, N. 2014, Planetary Exploration and Science: Recent Results and Advances (Berlin: Springer-Verlag)

Trifonov, T., Reffert, S., Tan, X., Lee, M. H., \& Quirrenbach, A. 2014, A\&A, 568, A64

Trifonov, T., Reffert, S., Zechmeister, M., Reiners, A., \& Quirrenbach, A. 2015, A\&A, 582, A54

van Leeuwen, F. 2007, A\&A, 474, 653

Welsh, W. F., Orosz, J. A., Carter, J. A., et al. 2012, Natur, 481, 475

Wisdom, J., \& Holman, M. 1991, AJ, 102, 1528

Wittenmyer, R. A., Tan, X., Lee, M. H., et al. 2014, ApJ, 780, 140

Zechmeister, M., Reffert, S., Hatzes, A. P., Endl, M., \& Quirrenbach, A. 2008, A\&A, 491, 531 